\title{
LHCb Upgrade: Scintillating Fibre Tracker
}

\author{
Mark Tobin \\ LPHE - EPFL
}

On behalf of the LHCb Upgrade SciFi Tracker group

Brazil (CBPF) - China (Tsinghua) - France (LPC, LAL, LPNHE) - Germany (Aachen, Dortmund, Heidelberg, Rostock)

Netherlands (Nikhef) - Russia (Kurchatov, ITEP, INR) - Spain (Barcelona, Valencia) - Switzerland (CERN, EPFL) 


\section{Overview}

\section{CERN-LHCC-2014-001}

- Introduction to $\mathrm{LHCb}$.

- Current detector.

- Upgrade detector.

- Scintillating Fibre Tracker.

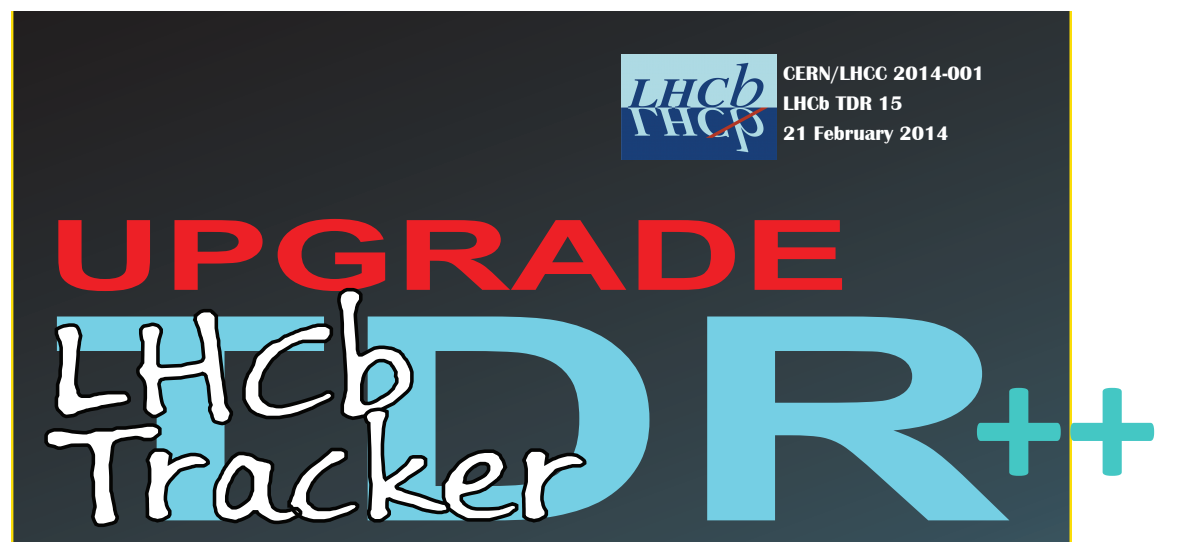

- Requirements.

- Scintillating fibres.

- Silicon photomultipliers.

- Electronics.

- Module production.

- Schedule.

- Conclusions.

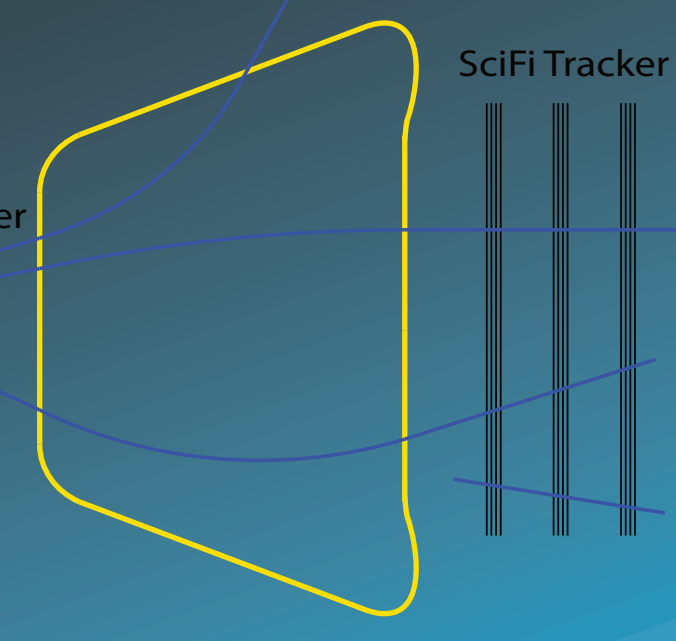




\section{Introduction}

\section{Current LHCb:}

- Described in talk by Albert Puig (today @ 12.20).

- Data taking conditions:

- Instantaneous luminosity $=4 \times 10^{32} \mathrm{~cm}^{-2} \mathrm{~s}^{-1}$.

- \# visible interactions / crossing, $\mu \approx 1.7$.

- Hardware trigger reduces rate from $40 \mathrm{MHz}$ to $1.1 \mathrm{MHz}$.

\section{LHCb Upgrade:}

- Upgrade conditions:

- Instantaneous luminosity $=2 \times 10^{33} \mathrm{~cm}^{-2} \mathrm{~s}^{-1}$ and $\mu=5.2$.

- Re-design some detectors to cope with higher occupancies.

- Full software trigger for every 25 ns bunch crossing.

- See talk by Antonio Falabella (28 ${ }^{\text {th }}$ May @ 16.50).

- Replace nearly all front-end electronics.

- Collect integrated luminosity $\approx 50 \mathrm{fb}^{-1}$. 


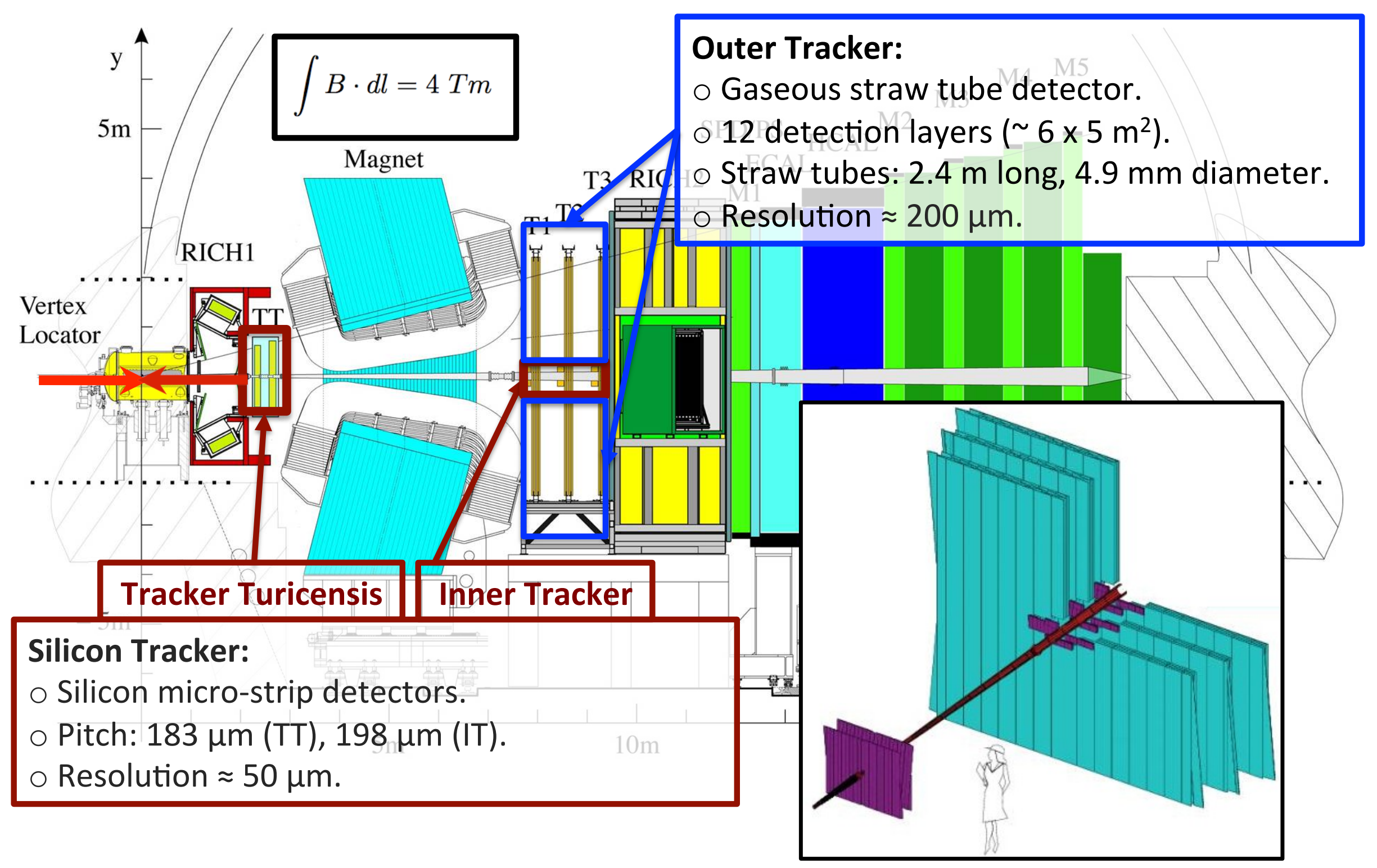




\section{Requirements on Upgrade Tracker}

- Detector performance:

- Hit efficiency $>98 \%$

- Noise rate $<10 \%$ signal rate (in same region).

- Resolution $\approx 100 \mu \mathrm{m}$ (in bending plane).

- Material: $X I X_{0} \leq 1 \%$ per layer.

- $40 \mathrm{MHz}$ read-out.

- Integrated luminosity $\approx 50 \mathrm{fb}^{-1}$. 


\section{- Redesign and replace IT+OT.}

- Occupancy too high in OT in upgrade conditions.

- Electronics designed for $1 \mathrm{MHz}$.

\section{- SciFi Tracker:}

- Scintillating fibres read out with Silicon Photomultipliers (SiPMs).

- Single fast and light technology.

- Read-out electronics and services outside acceptance.

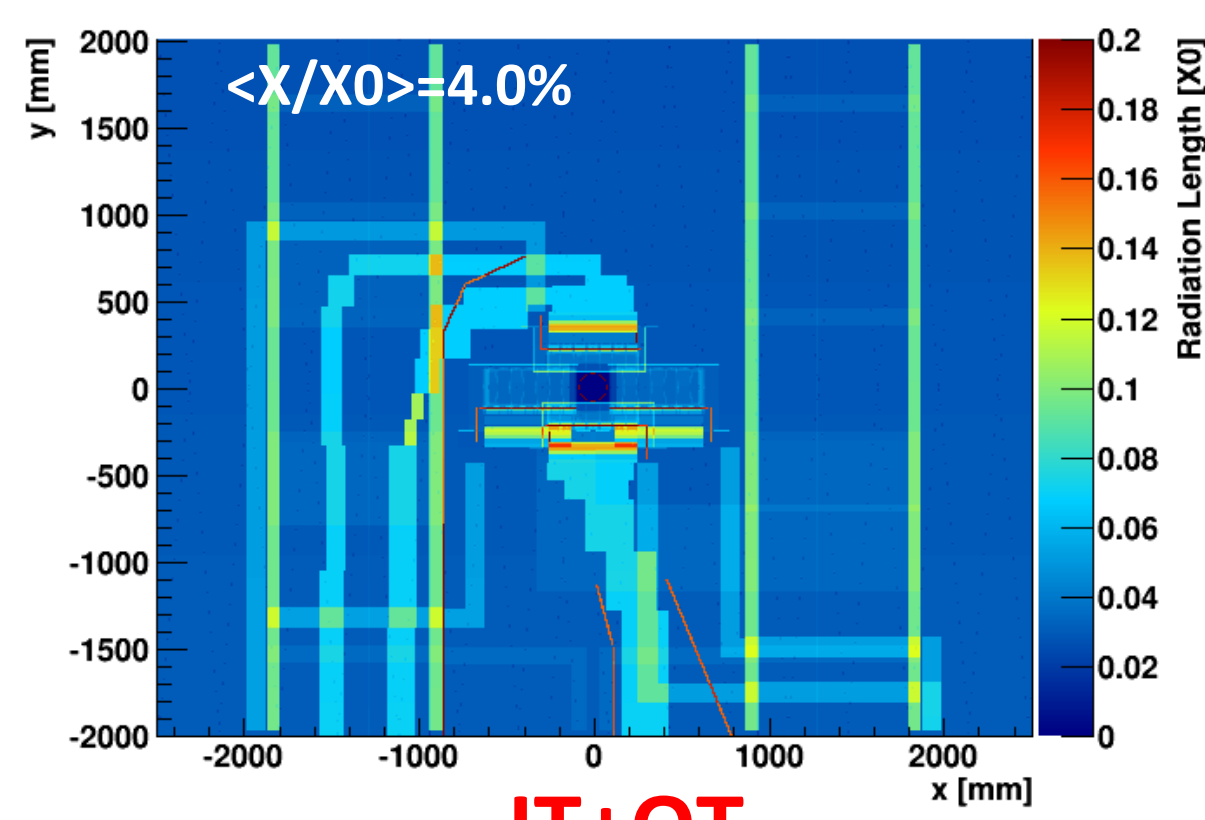

IT+OT

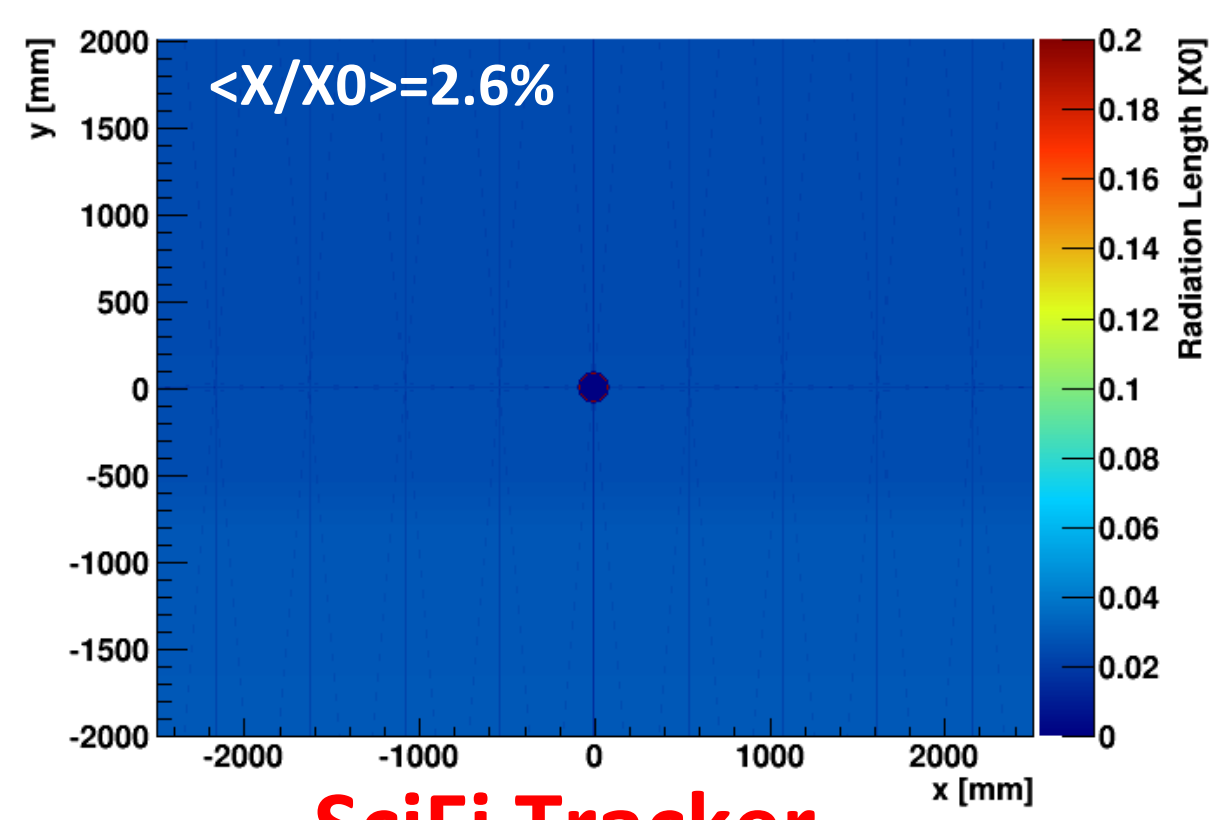

SciFi Tracker 
Detector design

- 12 modules per detection plane.

- Scintillating fibres.

- $2.4 \mathrm{~m}$ long, $250 \mu \mathrm{m}$ diameter.

- Mirrored at one end.

- Six layers of fibres in each module.

- 10,000 km needed for full detector.

- Total active area is $360 \mathrm{~m}^{2}$.

- SiPMs and front-end electronics

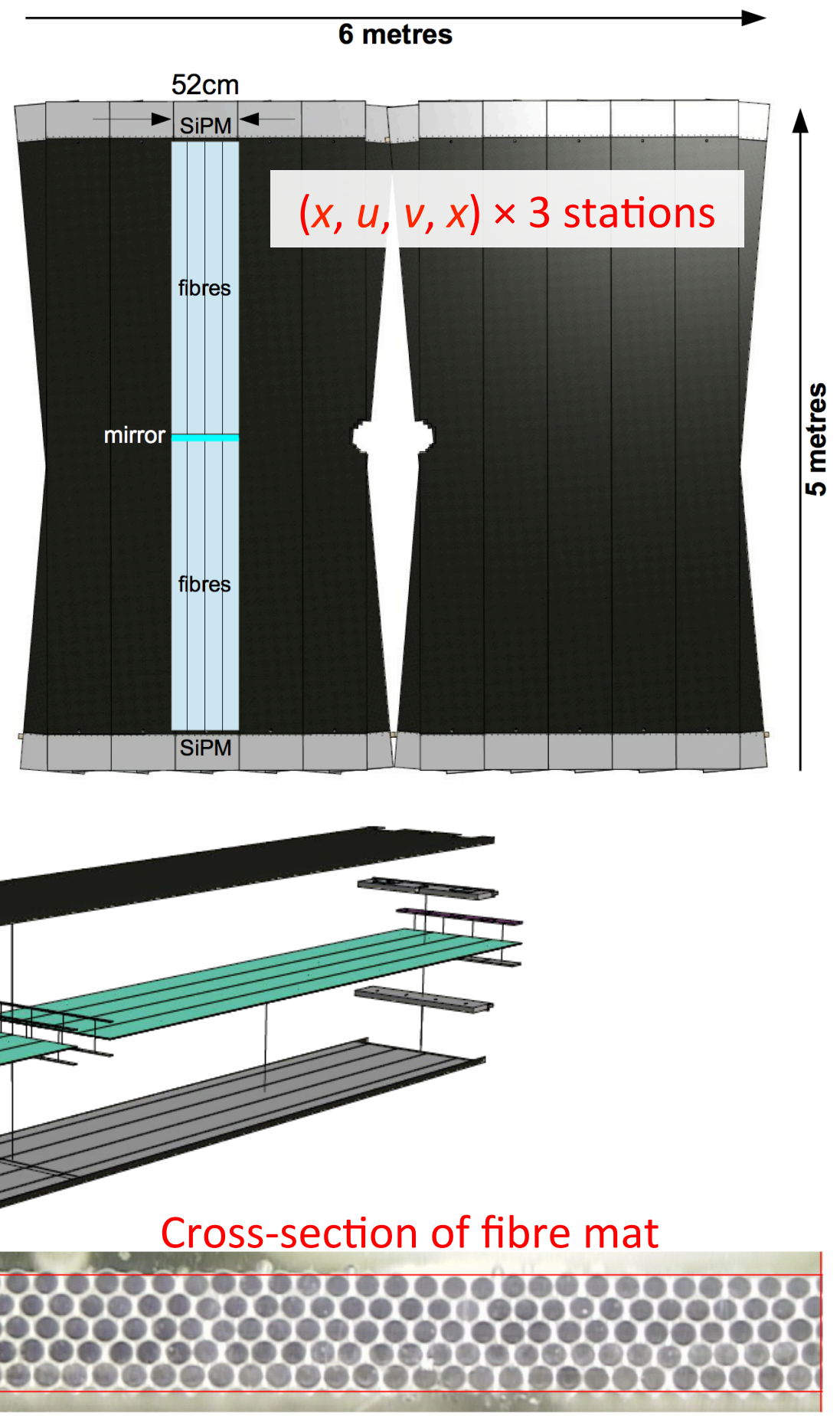




\section{$10,000 \mathrm{~km}$ (ish)}

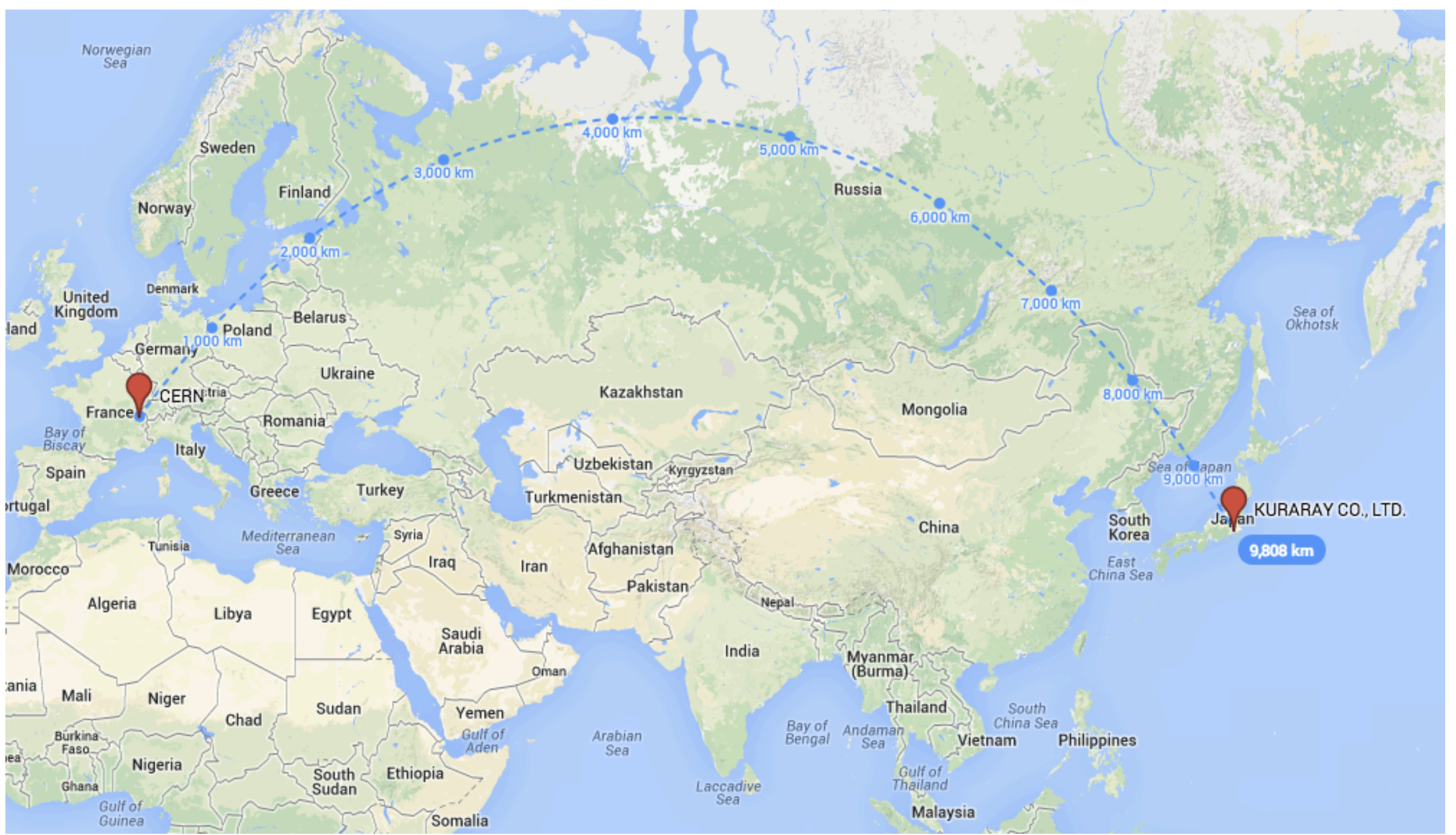




\section{Radiation environment}

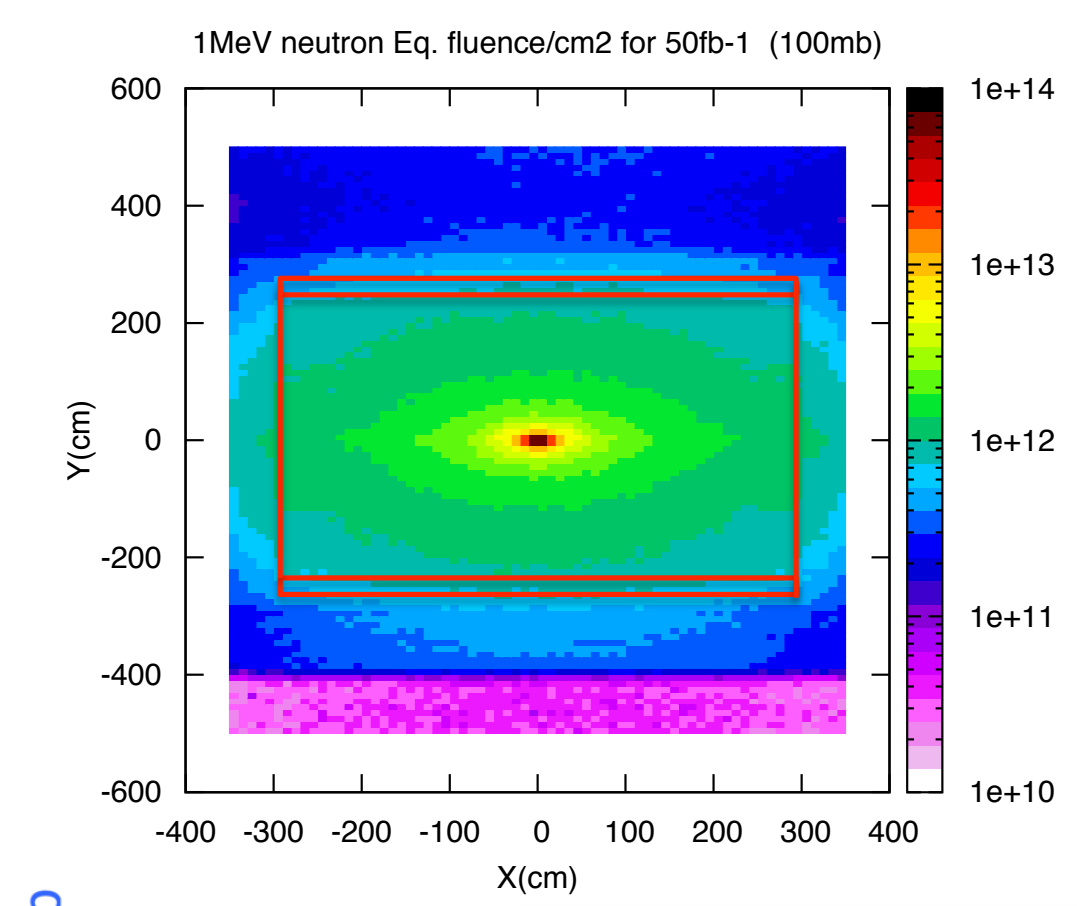

\section{NIEL (neutrons):}

- SiPMs at $\pm 250 \mathrm{~cm}$

$-9.5 \times 10^{11} \mathrm{n}_{\mathrm{eq}} / \mathrm{cm}^{2}$ (T1).

- $13 \times 10^{11} \mathrm{n}_{\mathrm{eq}} / \mathrm{cm}^{2}$ (T3).

- Shielding of SiPMs.

- Polyethylene with $5 \%$ boron.

$-6 \times 10^{11} \mathrm{n}_{\mathrm{eq}} / \mathrm{cm}^{2}$.

\section{lonising dose:}

- $35-25$ kGy (fibres).

- 40-80 Gy (SiPMs).
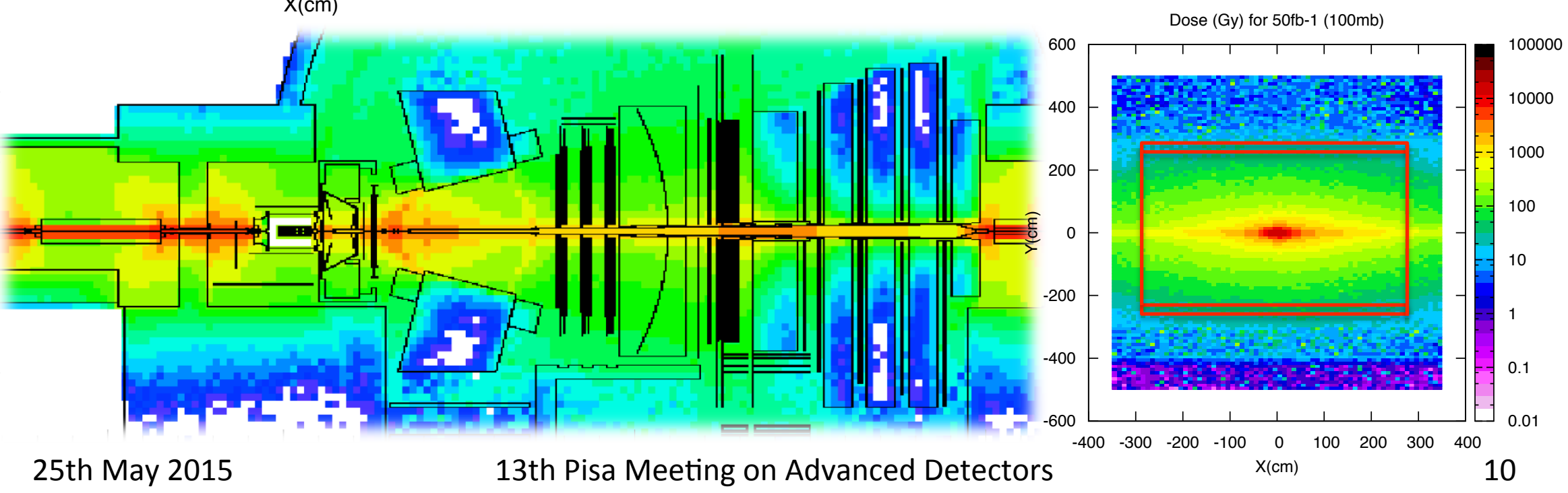


\section{Challenges}

- Procurement of scintillating fibres:

- High light yield.

- High attenuation length.

- Radiation hardness of fibres \& SiPMs.

- Mechanical design and construction.

- Fast read-out / data reduction.

- Cooling requirements.

- Timescale.

- Installation in Long Shutdown 2 (2018/19). 
Baseline fibre: Kuraray SCSF-78MJ

Total Internal Reflection

at First/Second Cladding Interface

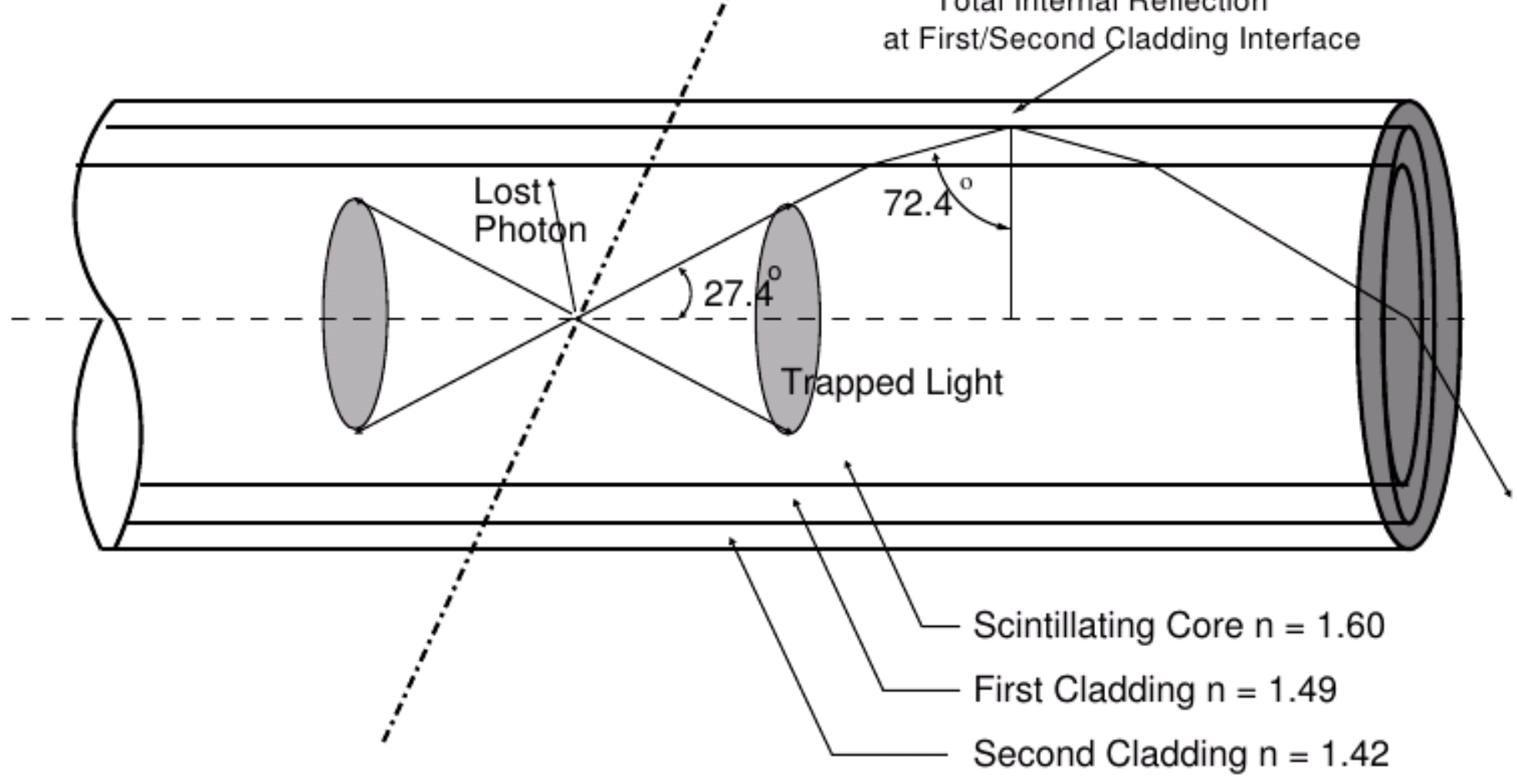

- Polystyrene core with two wavelength shifting dyes.

- Around 300 photons / MIP.

- Only a few photons after $2.4 \mathrm{~m}$.

- Fibres must have long attenuation length $(>300 \mathrm{~cm})$. 


\section{Irradiation effects in fibres}

Pre-irradiation

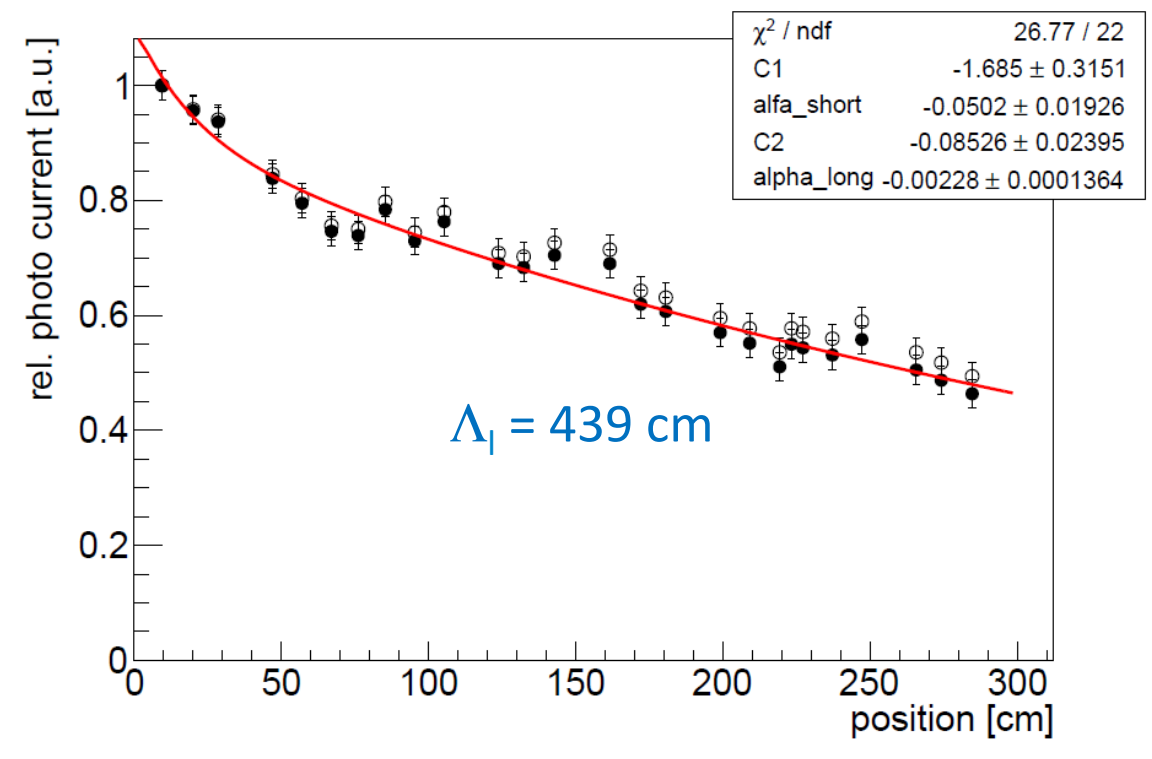

After irradiation

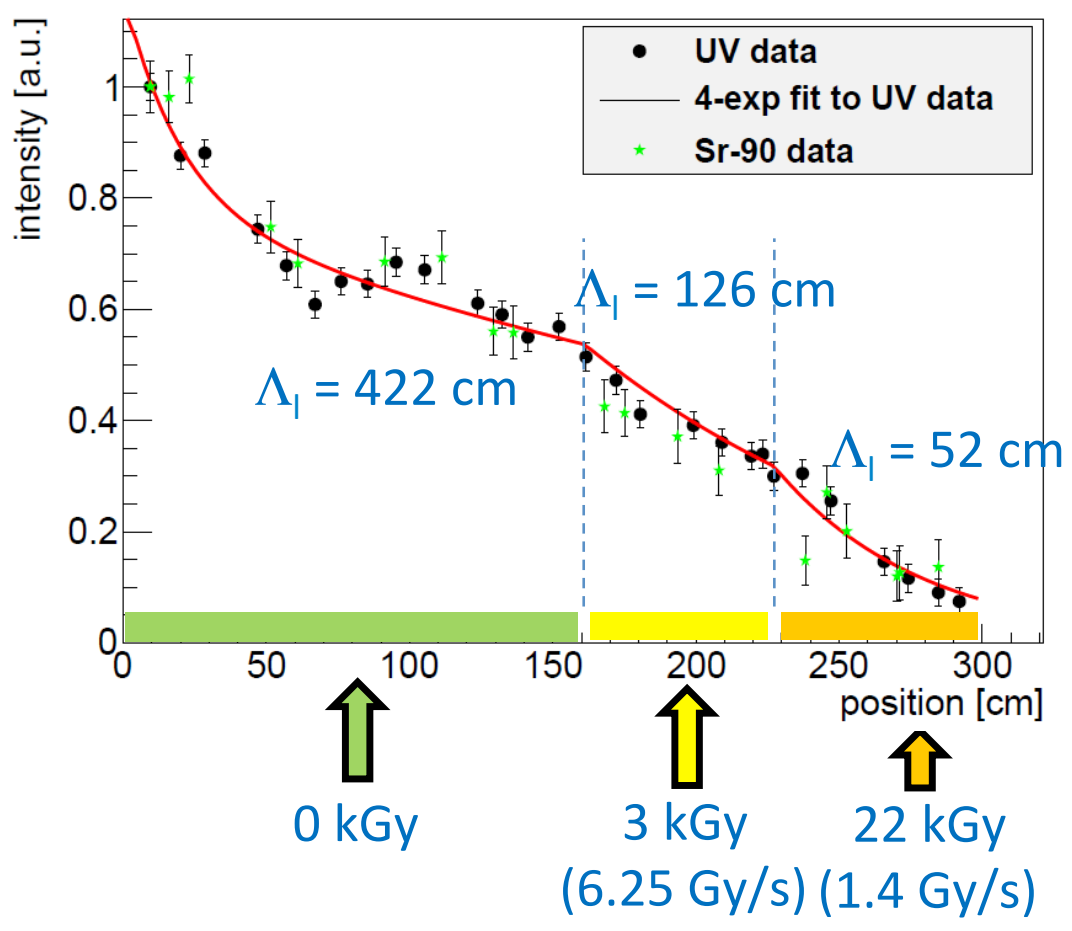

- Irradiation test at CERN PS with $24 \mathrm{GeV}$ protons.

- $3 \mathrm{~m}$ long SCSF-78 fibres ( $\varnothing 0.25 \mathrm{~mm}$ ).

- Embedded in glue (EPOTEK H301-2).

- Other irradiations with X-ray, gamma, electrons. 


\section{Silicon} Photomultipliers

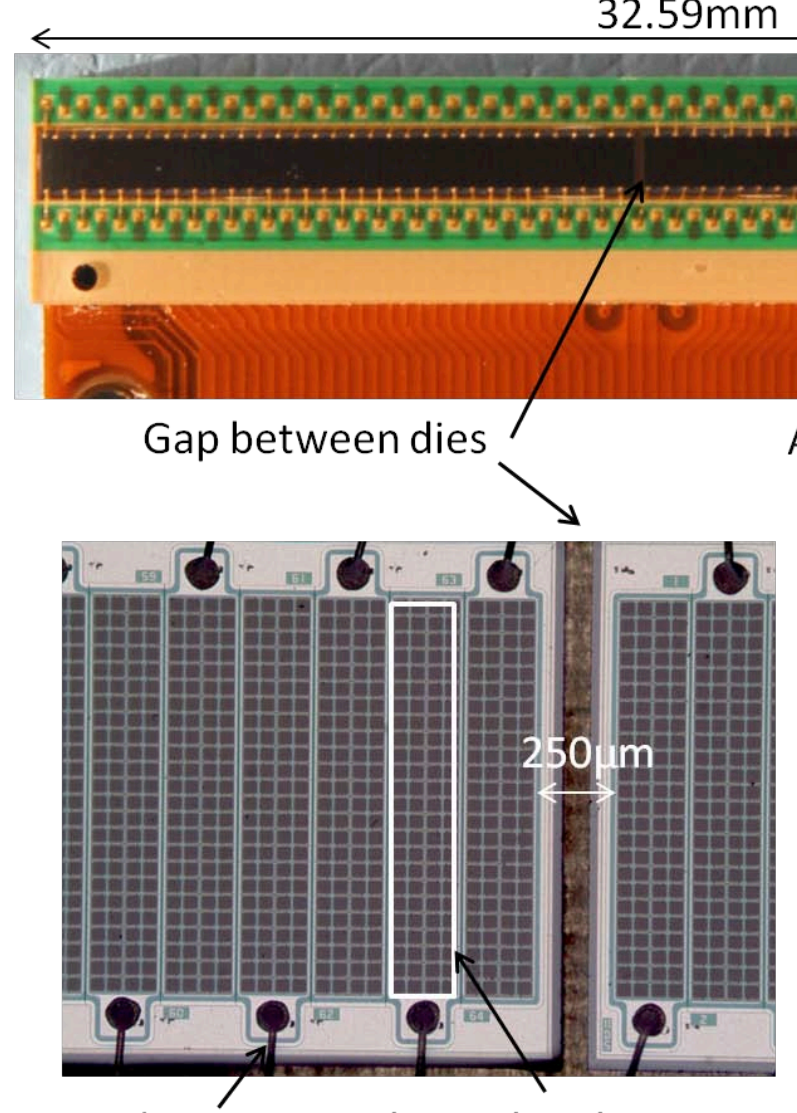

Bonding wire Channel outline

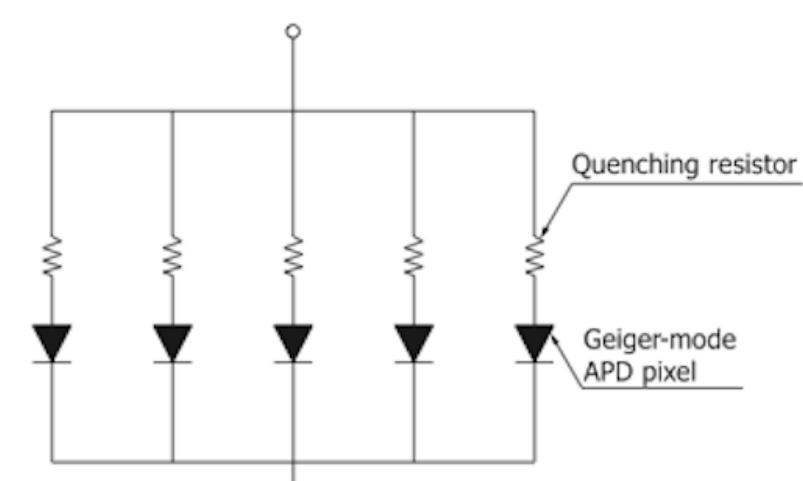

Active area

Active area

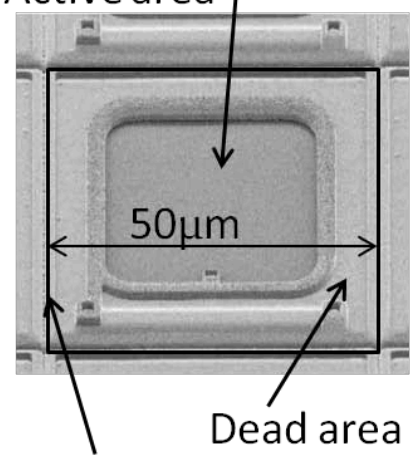

Pixel outline
- 128 channels / array.

- 100 pixels connected in parallel /channel.

- Avalanche photo-diode operated in Geiger mode.

- Channel width: $250 \mu \mathrm{m}$.

- 147m instrumented:

- 590k channels.

- 4608 SiPMs.

- Connected to read-out electronics via flex PCB.
Co-development with:

- Hamamatsu (Japan).

- KETEK (Germany).
Many different devices:

- Various pixel sizes.

- With/without trenches. 


\section{Characterisation of SiPMs}
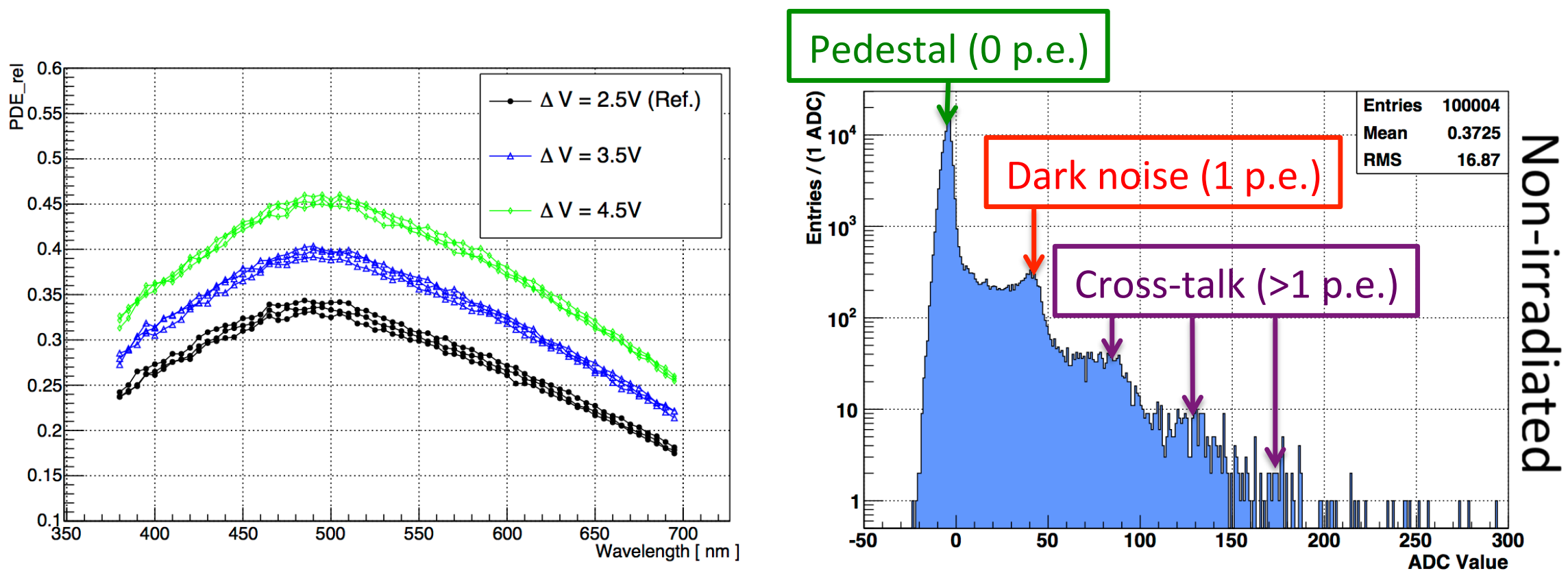

- High gain: $10^{6}-10^{7}$.

- High PDE at $\Delta \mathrm{V}=3.5 \mathrm{~V}$ !

- 39\%@ 490 nm.

- Dark count rate.

- Cross-talk.

- Temperature dependent.

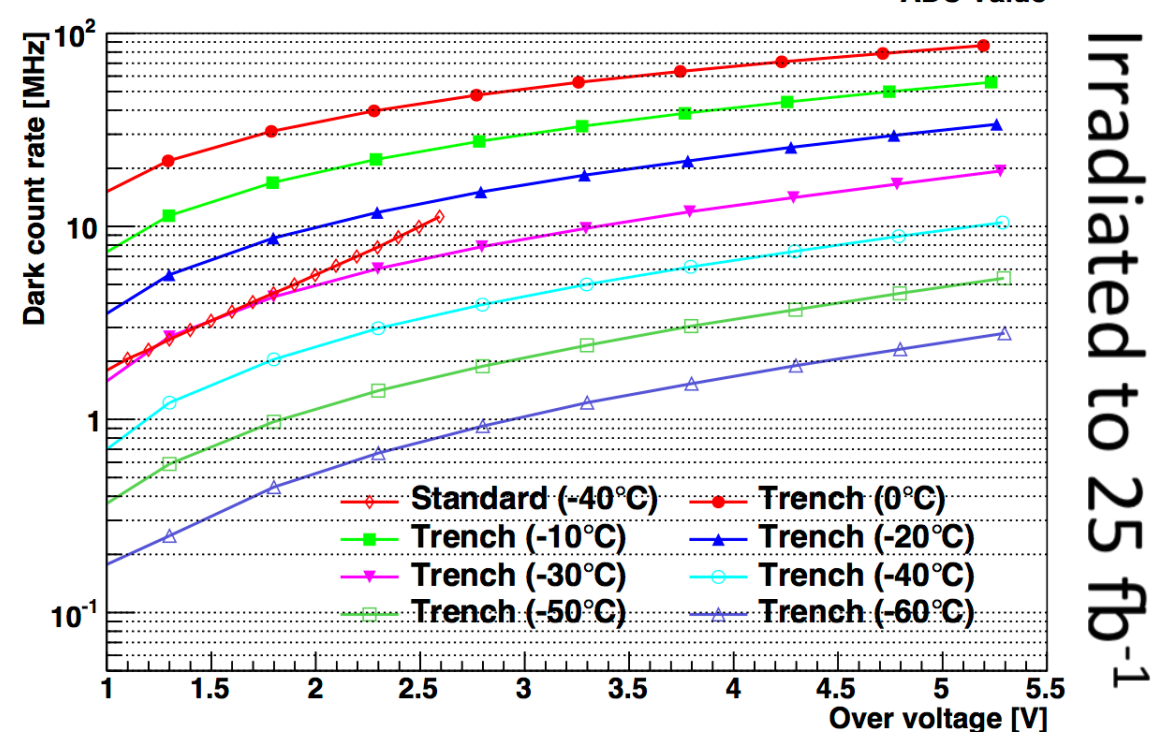




\section{Fibres:}

- Emission spectra.

- Measured at different distances from detector.

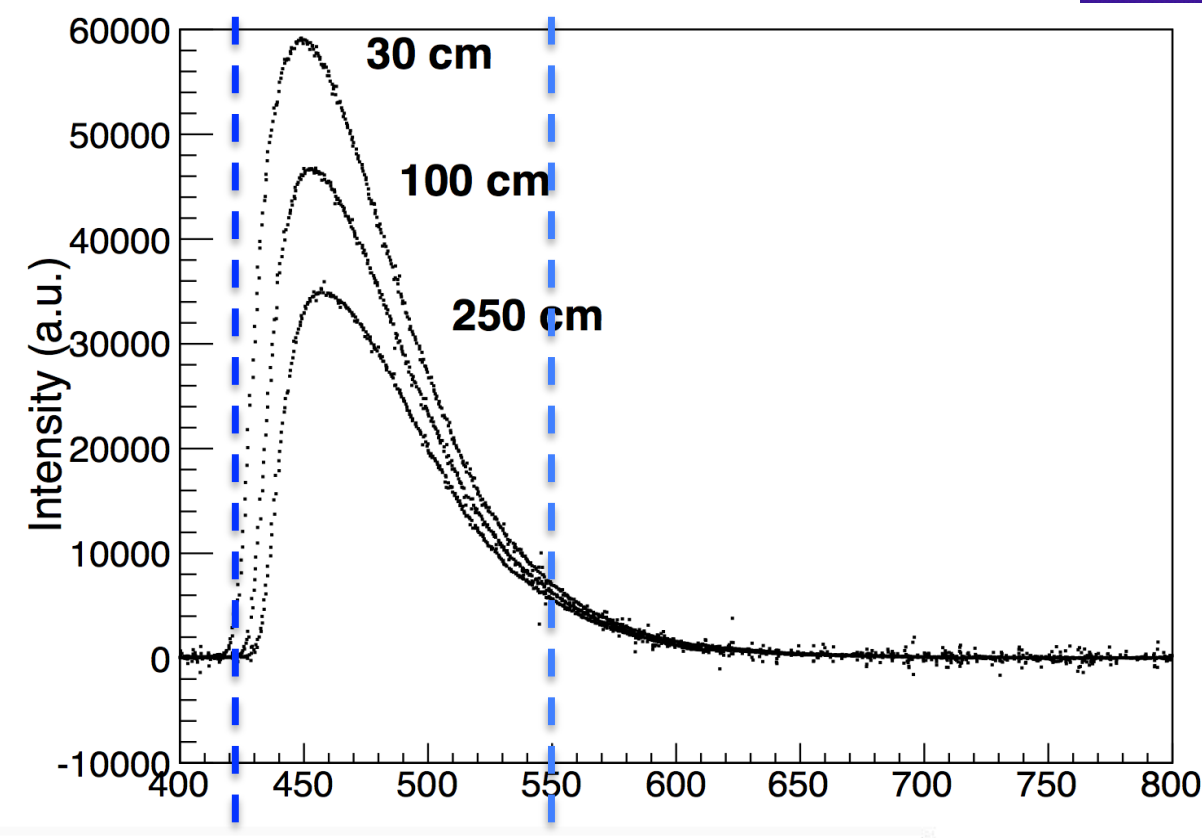

\section{SiPMs:}

- $\mathrm{PDE}=\mathrm{QE} \times \mathrm{GF} \times \varepsilon_{\mathrm{AT}}$

- PDE: Photon detection efficiency.

- QE: Quantum Efficiency.

- GF: Geometrical factor.

- $\varepsilon_{\mathrm{AT}}$ : Avalanche trigger probability.

- Single channel device.

- $50 \mu \mathrm{m}$ pixels.

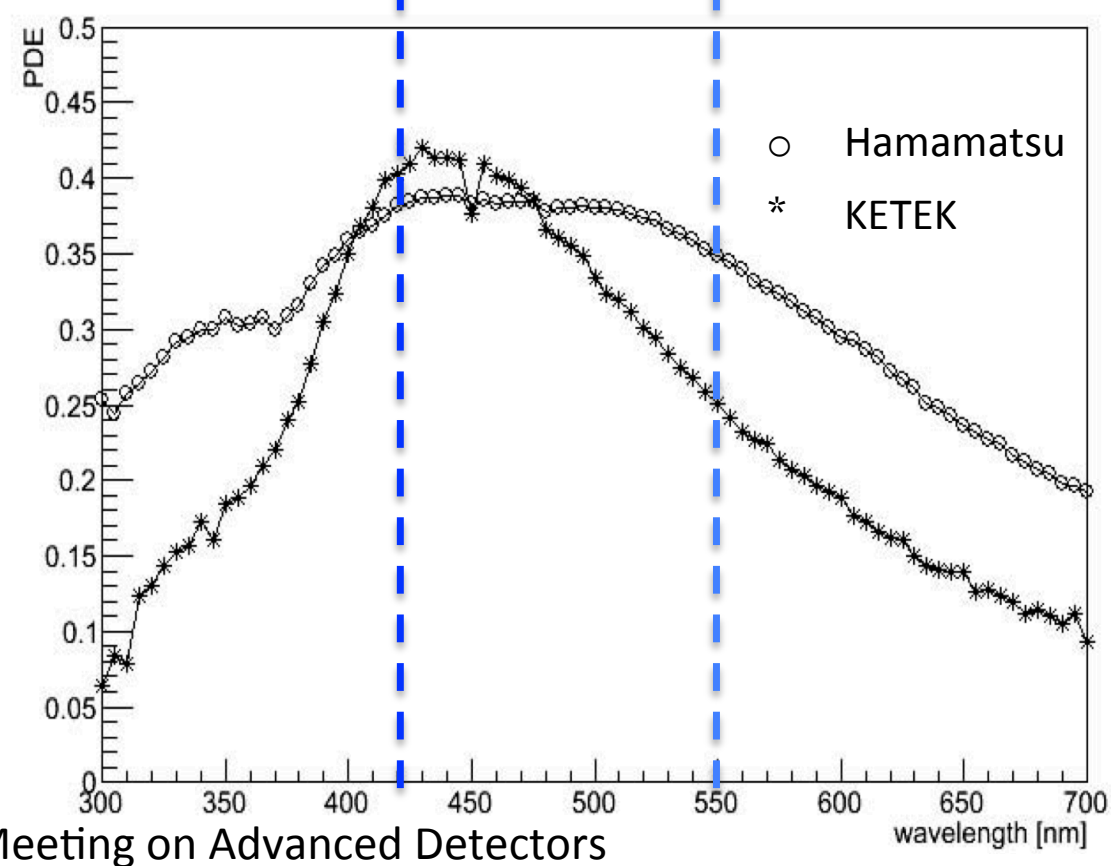




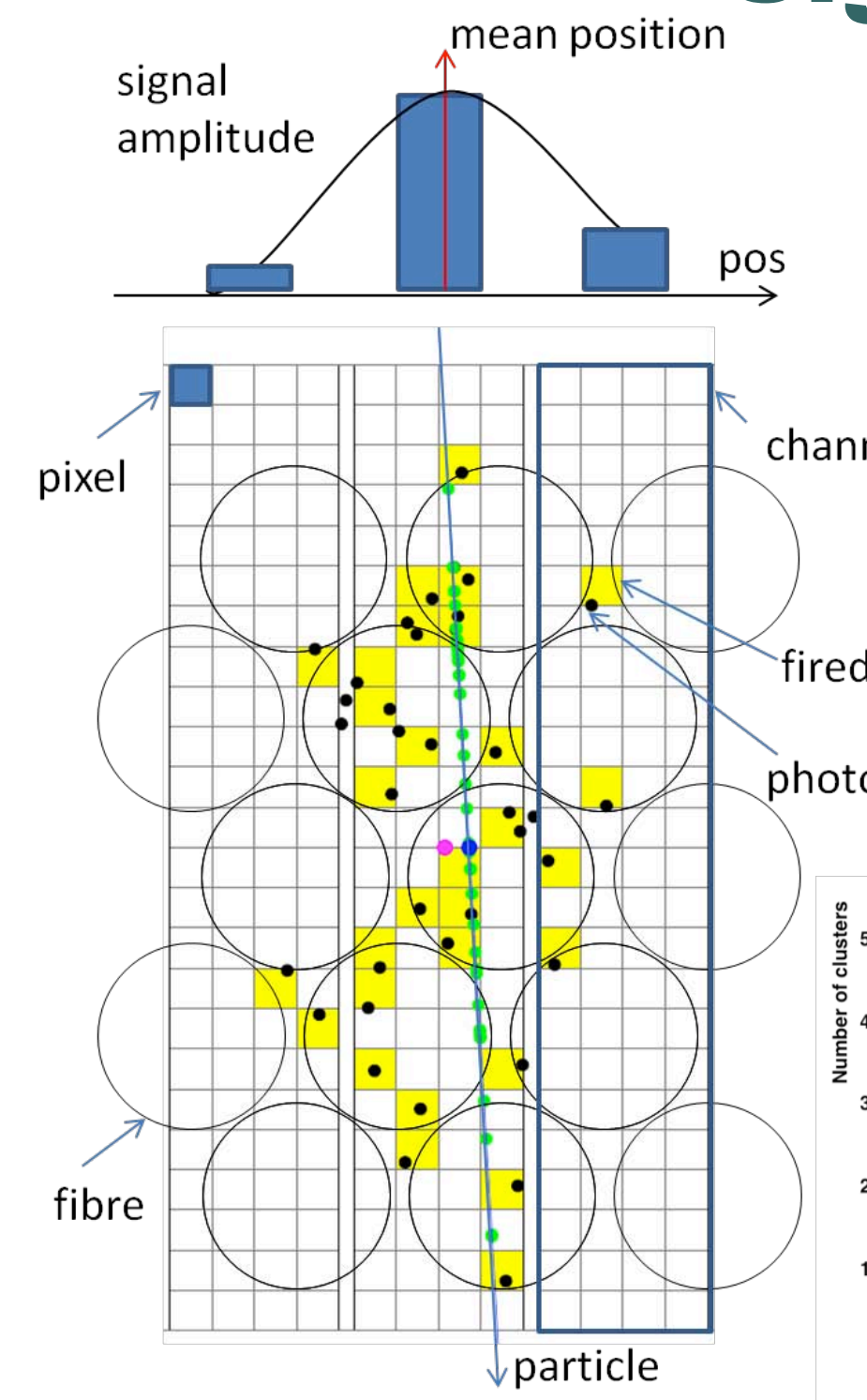

Channel pitch < fibre pitch.

\section{Clusters:}

- Signals shared over two (or more) channels. SiPMs:

- Dark noise increases with neutron radiation.

- Cooling and annealing.

\section{Fibres:}

- Darken after irradiation.

- Six layers of fibres to maximise light yield. Expected signal:

- 12 - 16 photo-electrons after irradiation.
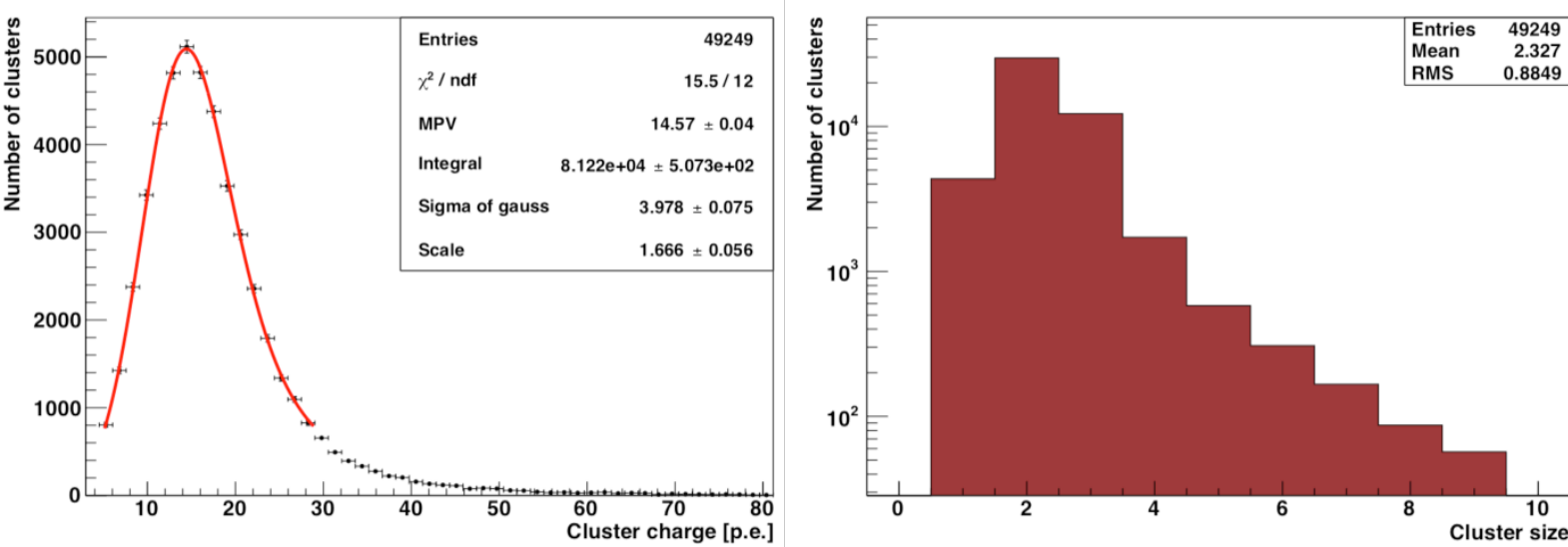

Cluster charge and size measured in cosmic ray telescope. 


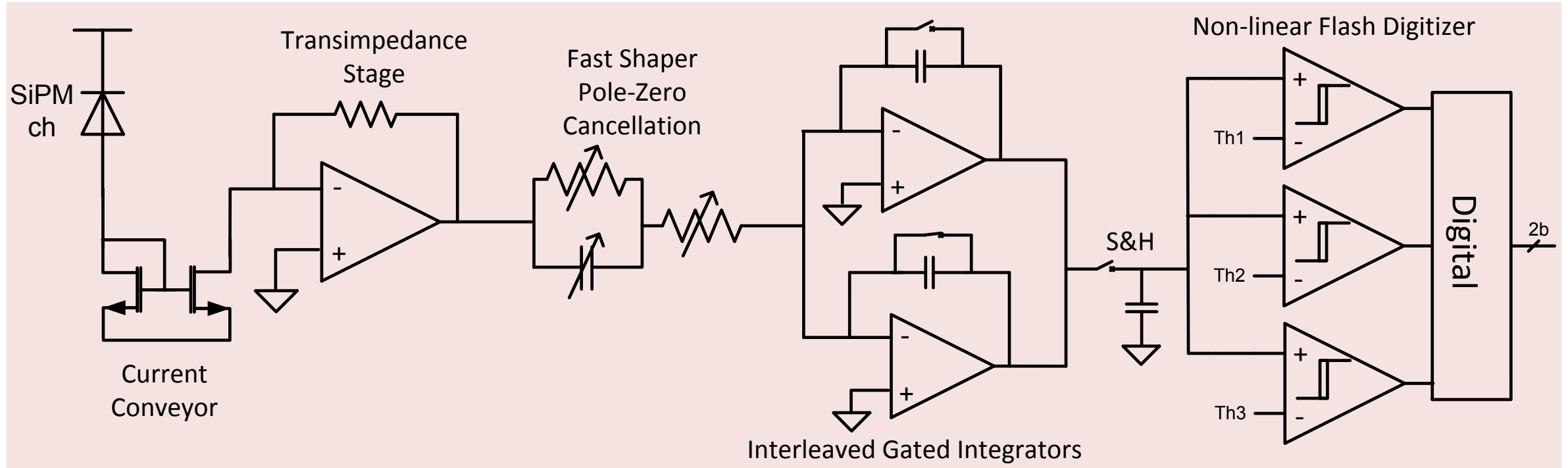

- Trigger-less read-out.

- Zero-suppression in front-ends.

- Development of custom front-end ASIC.

- PACIFIC:

- 64 channels, 130 ns CMOS (TSMC).

- Three hardware thresholds = 2-bit.

- Bandwidth: $300 \mathrm{MHz}$.

- Low input impedence: 20-40 $\Omega$.

- Low power $8 \mathrm{~mW} /$ channel.

- Fast shaping with zero dead-time.

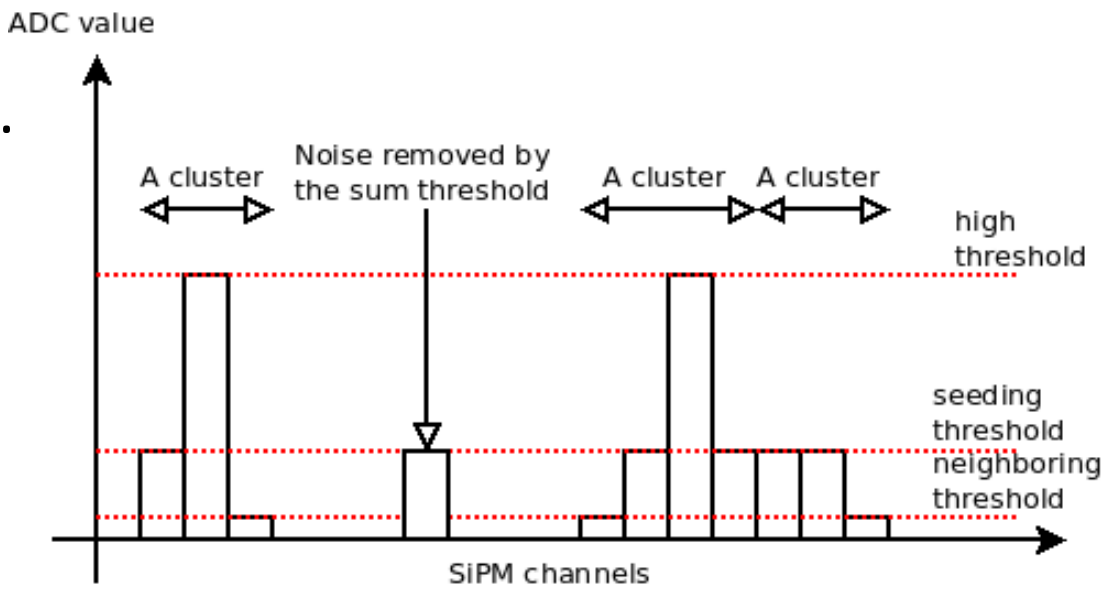

Clustering on FPGA (sum threshold). 


\section{Read-out Box}

- Every module has one read-out box at each end.

- Light tight and gas tight.

- Ensures precise optical coupling of cold $\left(-40^{\circ} \mathrm{C}\right) \mathrm{SiPMs}$ to fibres.

- Houses warm front-end electronics.

- Provide mechanical coupling to support structure.

Cold part of box (illustrative). Coolant: C6F14 or Novec 649. Heat load: $20 \mathrm{~W} /$ box.
Hybrid connectors

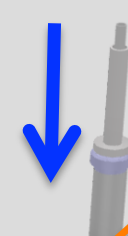

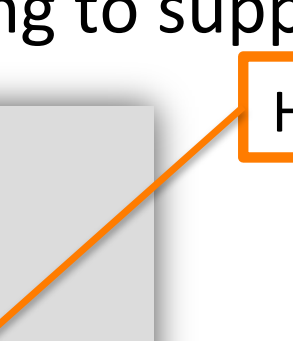

\section{.}
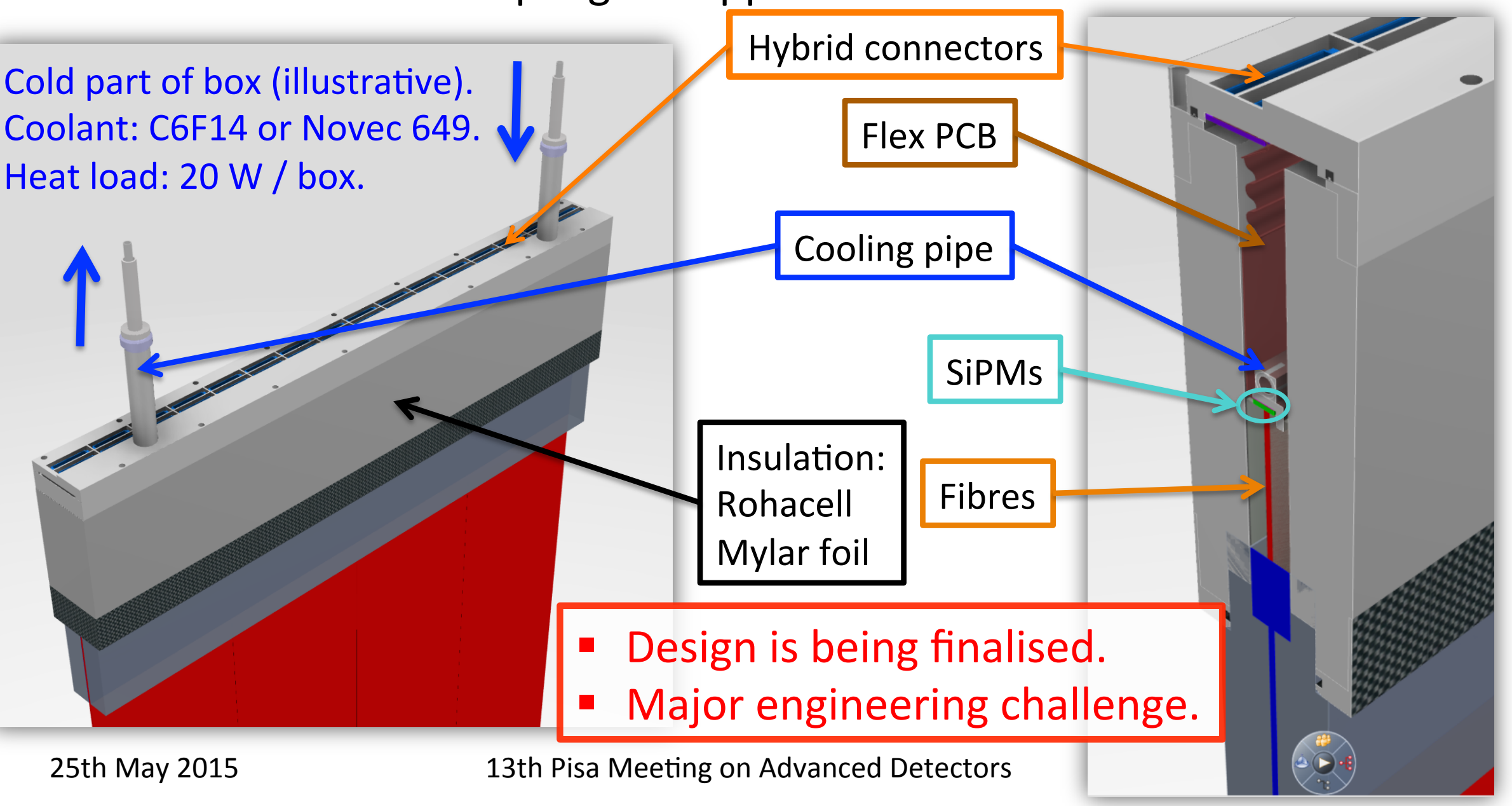


\section{Test beam}

LHCb
HHCP

- Test beam at CERN SPS:

- October / November 2014.

- May 2015 - ends at 7am tomorrow!

- Goals:

- Attenuation length.

- Hit efficiency.

- Resolution.

- Compare with measurements made in the lab and with the simulation.

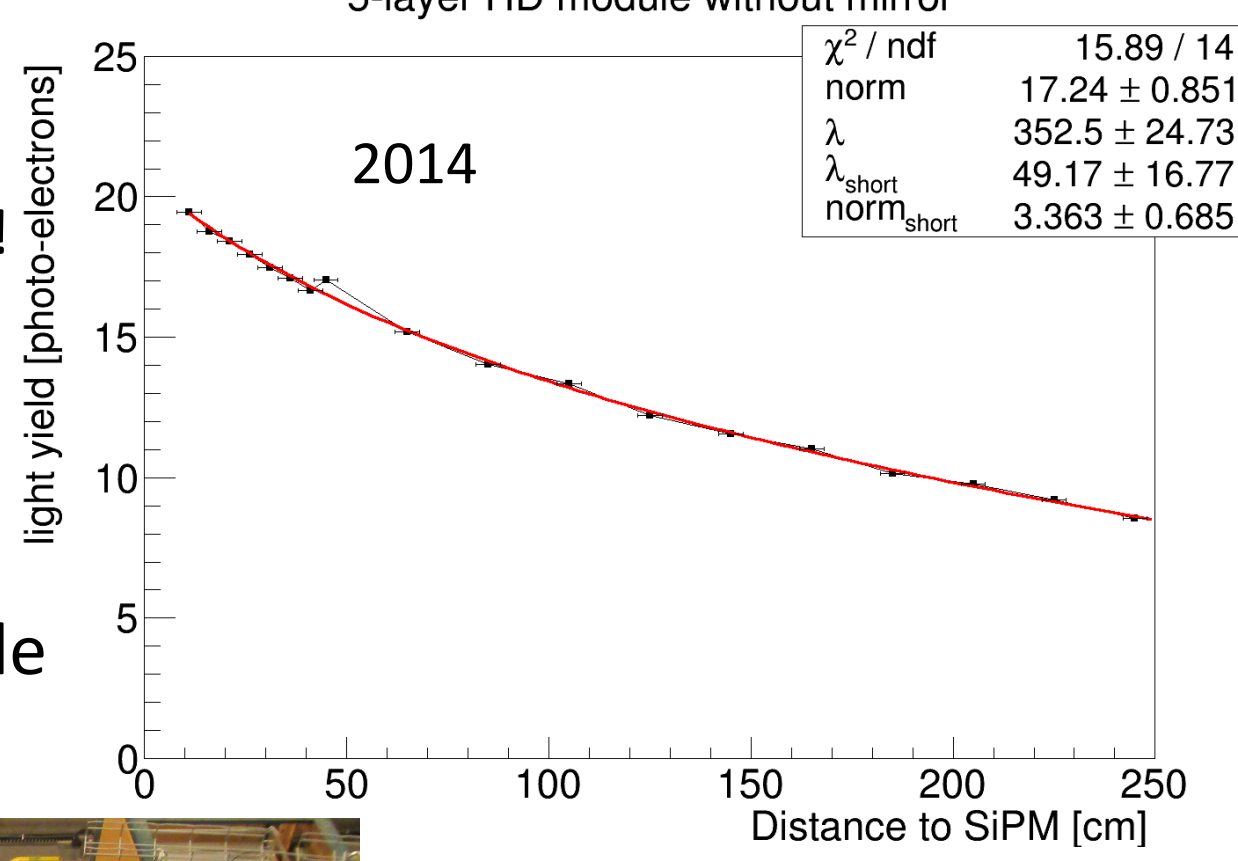

- Four DUTs.

- 5 and 6 layer mats.

- 2.5 m long.

- Hamamatsu SiPMs.

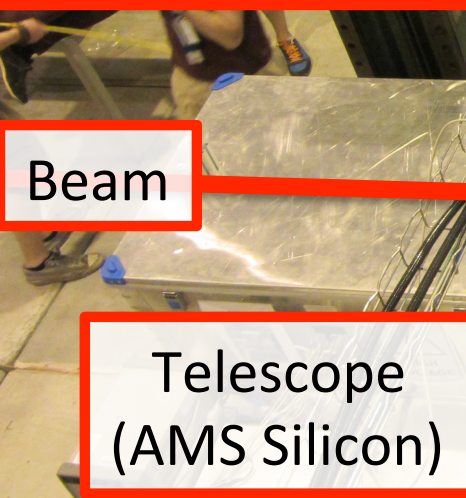

Beam

Telescope (AMS Silicon)

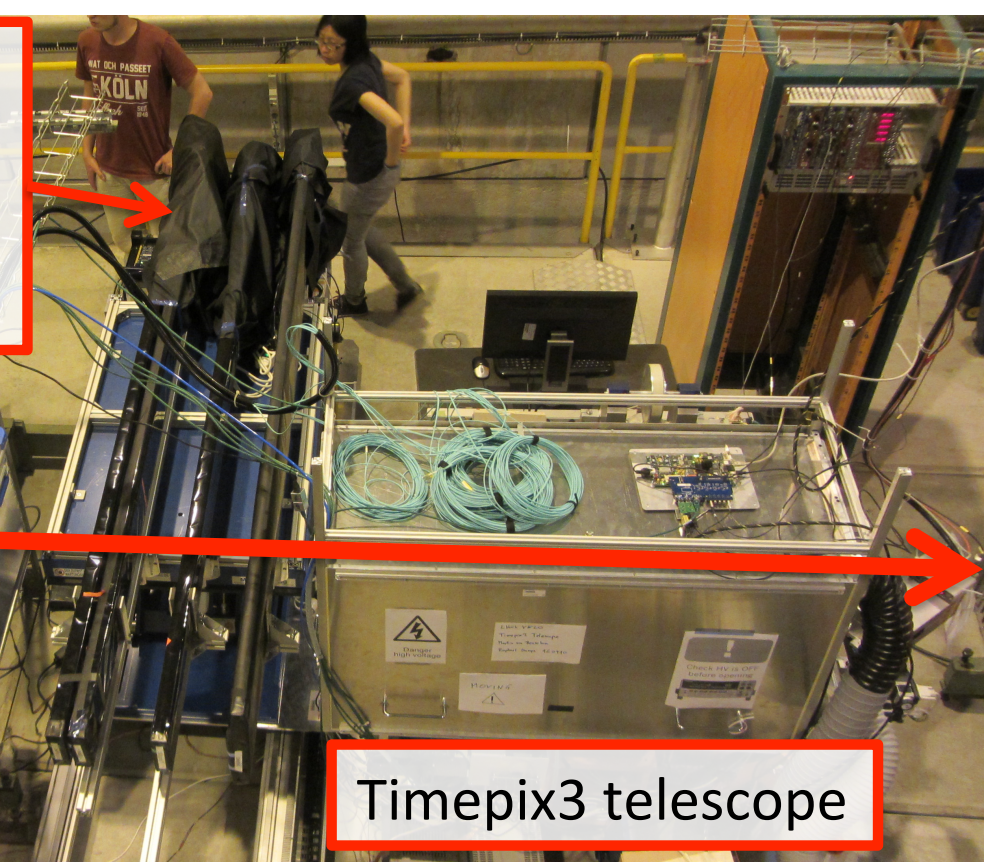

Distance to SiPM [cm]

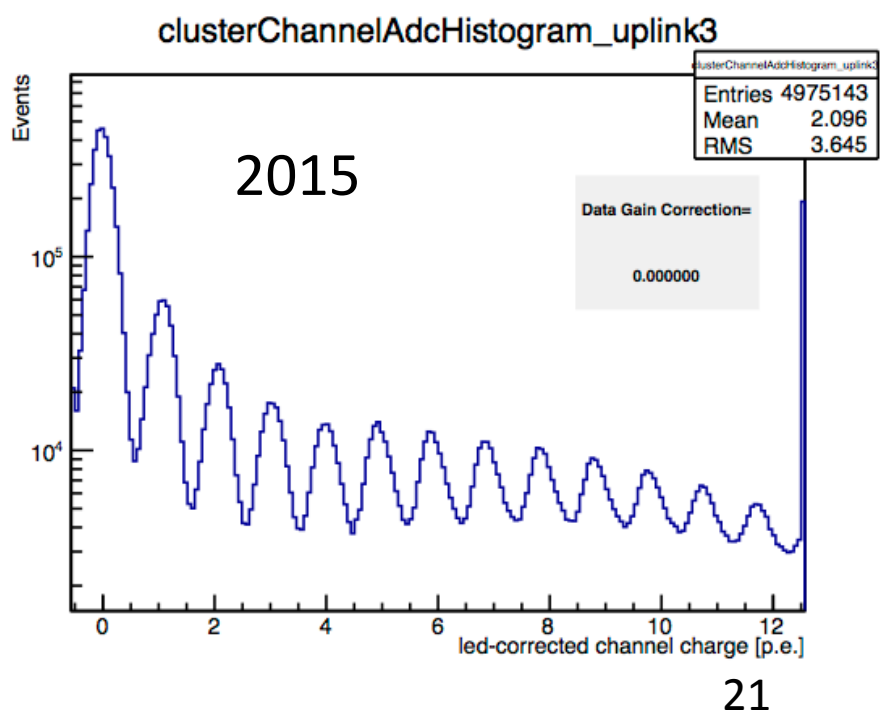




\section{Schedule / timeline}

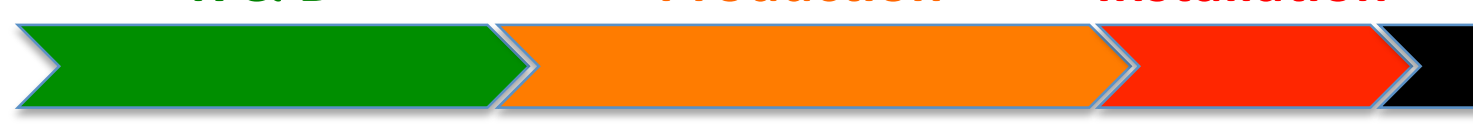

(More) New Physics?

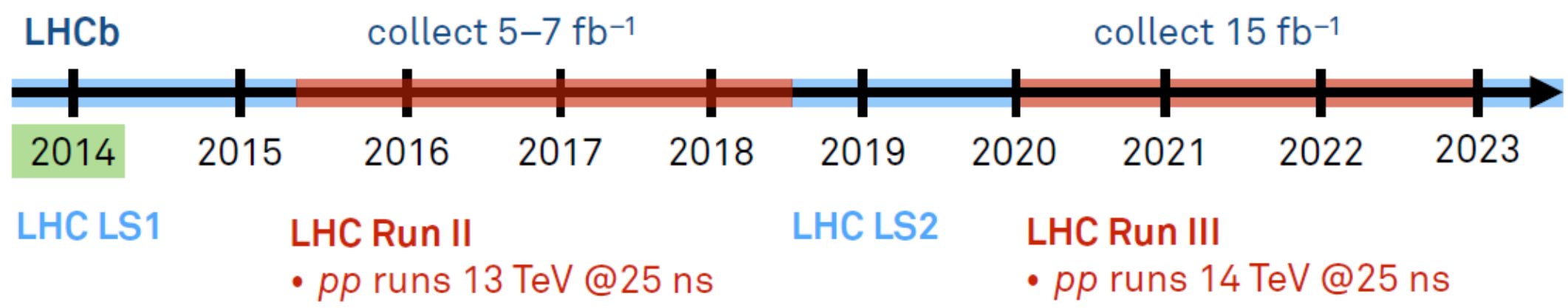

- Installation during LS2.

- Collect $50 \mathrm{fb}^{-1}$ after upgrade. 


\section{Conclusions}

LHCb Upgrade:

- Instantaneous luminosities up to $2 \times 10^{33} \mathrm{~cm}^{-2} \mathrm{~s}^{-1}$.

- Trigger-less read-out system.

- New tracking system with scintillating fibres.

\section{SciFi Tracker:}

- Scintillating fibres produced by Kuraray.

- High attenuation length and light yield.

- Silicon photomultipliers:

- Co-development with Hamamatsu and Ketek.

- High PDE and low cross-talk.

- Radiation damage studied up to $50 \mathrm{fb}^{-1}$.

- Six layers of fibres to ensure sufficient light yield.

- Shielding required for SiPMs.

- Module production will start at end of 2015. 


\section{Frontier Detectors} for Frontier Physics $13^{\text {th }}$ Pisa meeting on advanced detectors

La Biodola • Isola d'Elba • Italy May 24 - 30, 2015

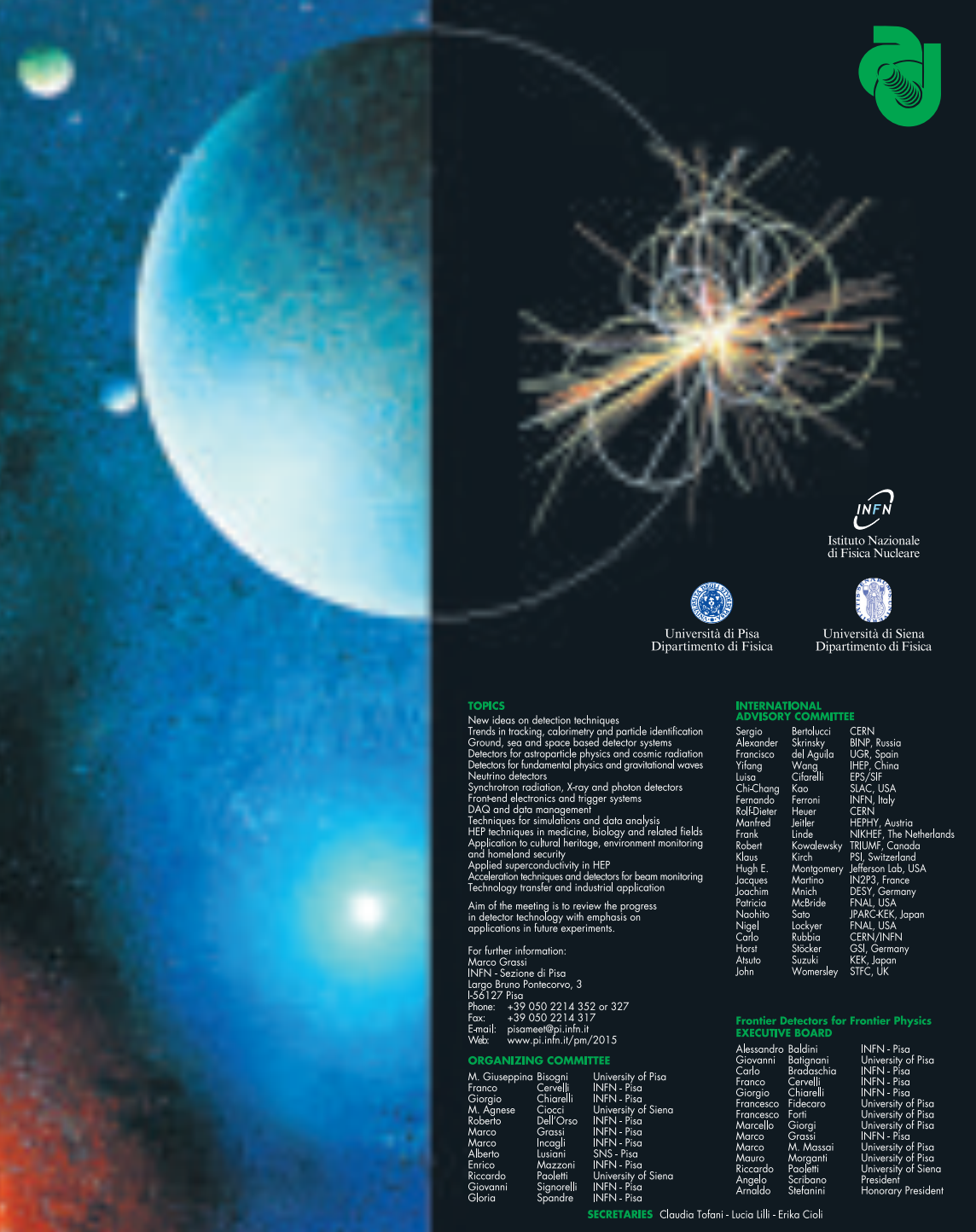

\section{Grazie mille!}




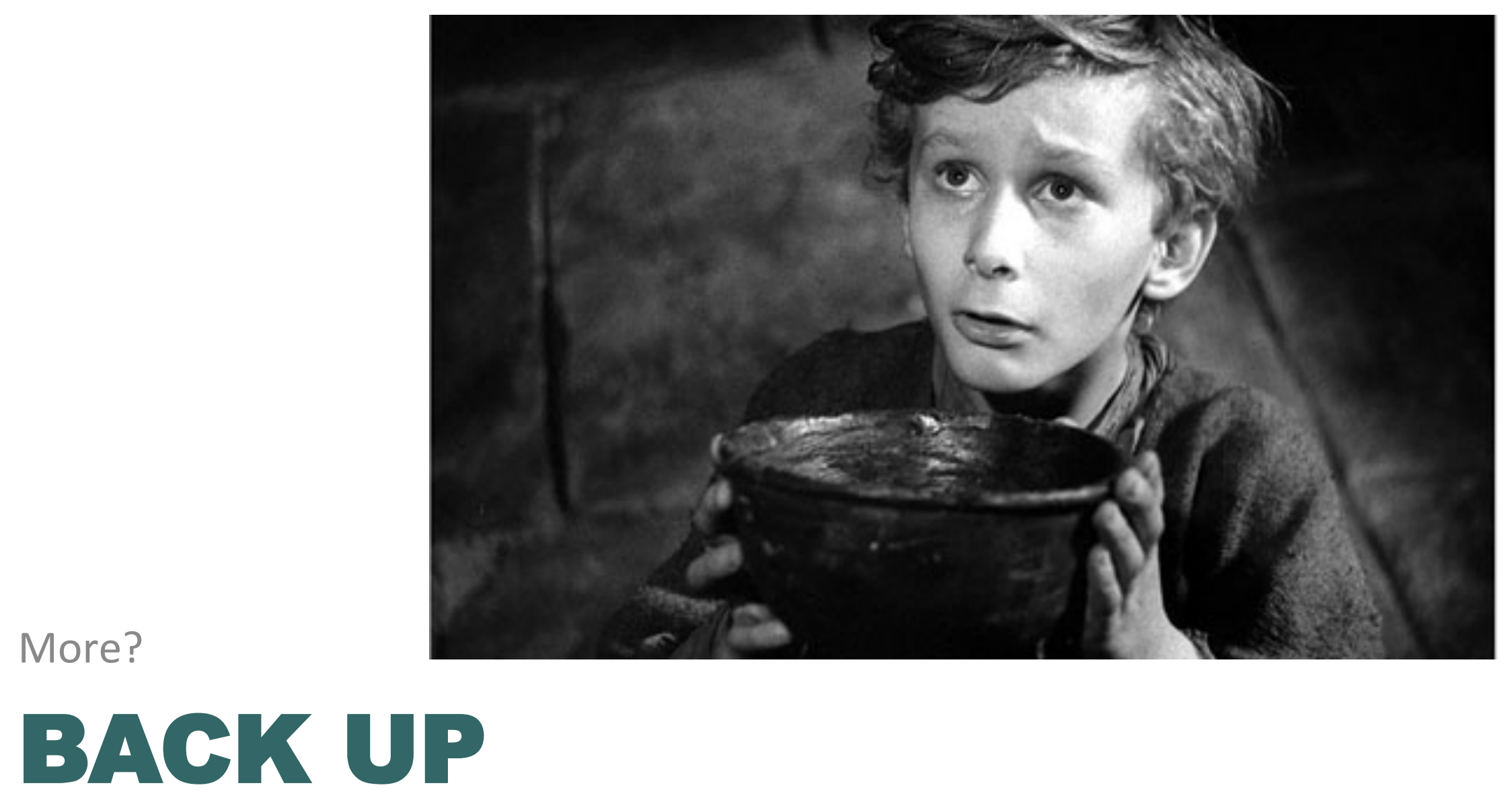




\section{Why upgrade?}

- No evidence for New Physics in LHC Run 1.

- Look for deviations from Standard Model.

- Most measurements still limited by statistics.

- Limited by Level-0 hardware trigger.

- Maximum rate is $1.1 \mathrm{MHz}$.

- Increase luminosity:

- Already ran well above design.

- Trigger yield saturates.

- No real gain in statistics.

- Higher occupancy.

- Degraded detector performance.

- Radiation damage of detectors.

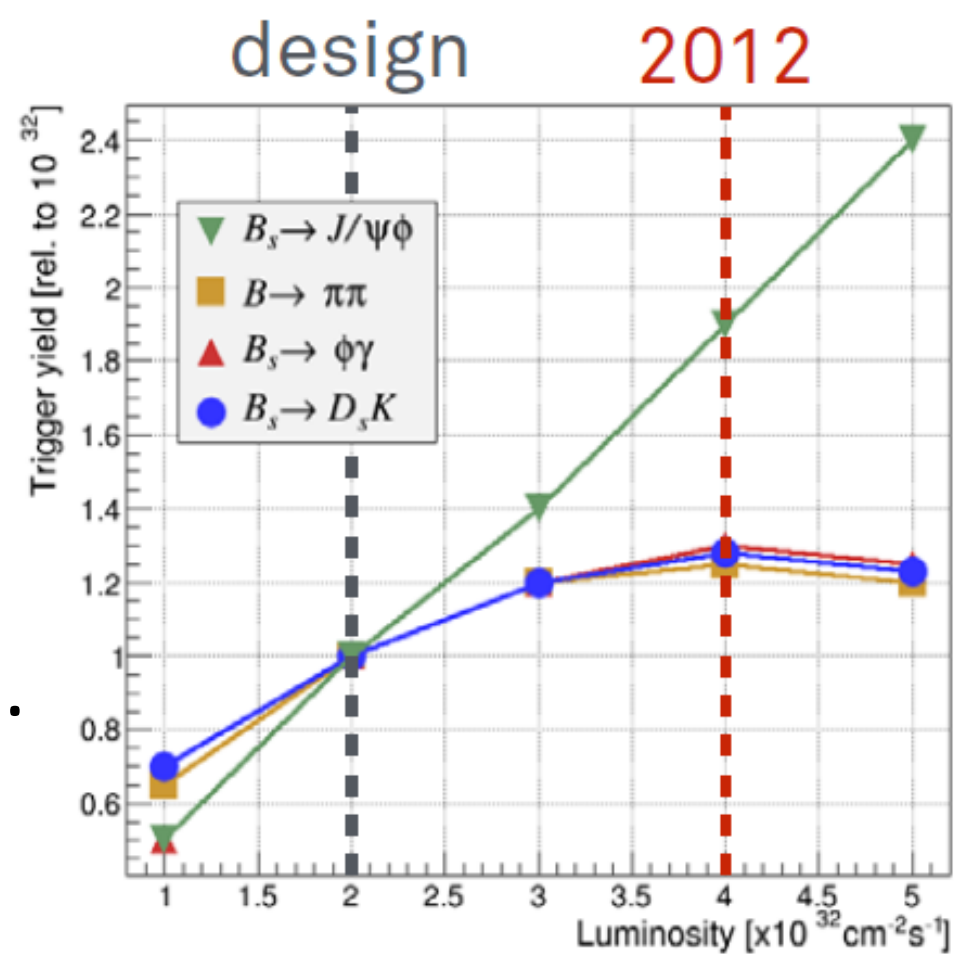




\section{Fibre diameter}
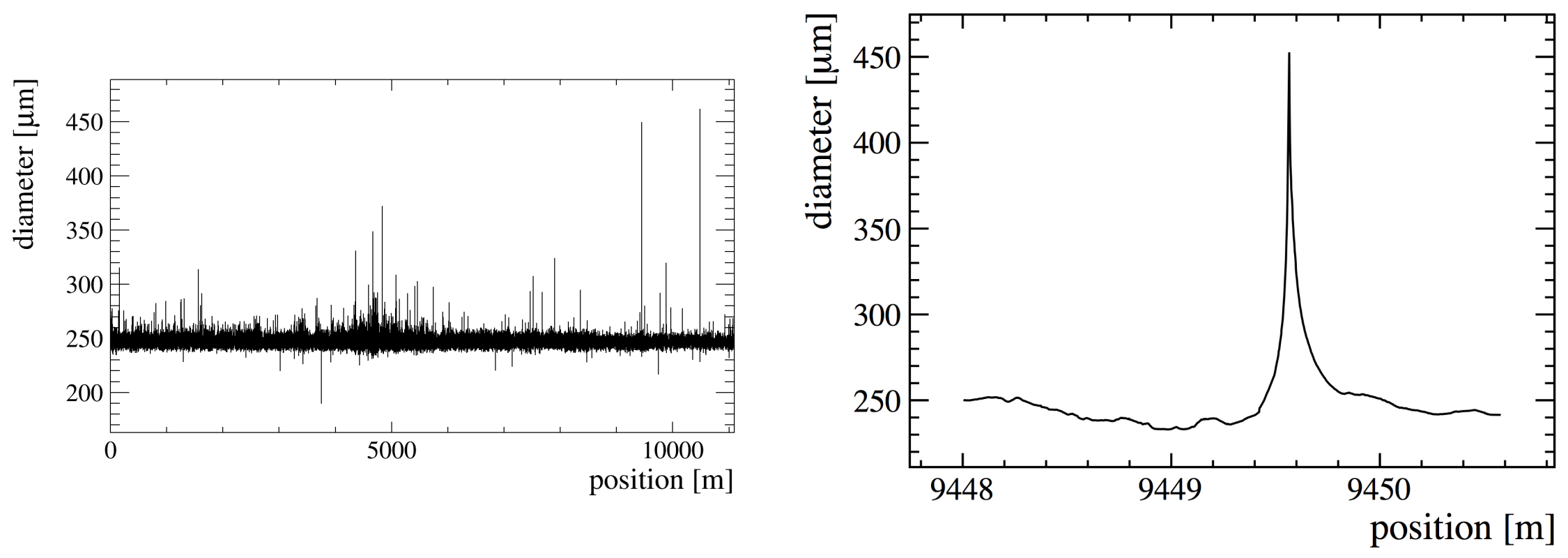

- Measure diameter every $3 \mathrm{~mm}$ with laser micrometer.

- Once per km, diameter goes above limit (300 $\mu \mathrm{m})$.

- Manually remove during winding process. 


\section{Radiation damage in fibres}

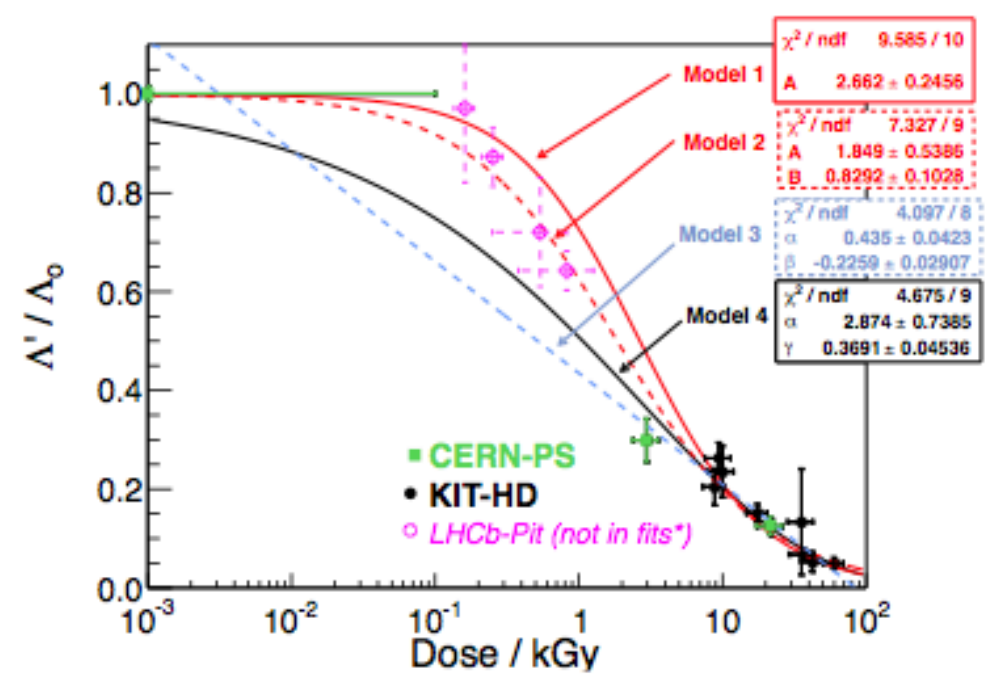

(a) Logarithmic $\mathrm{x}$-axis

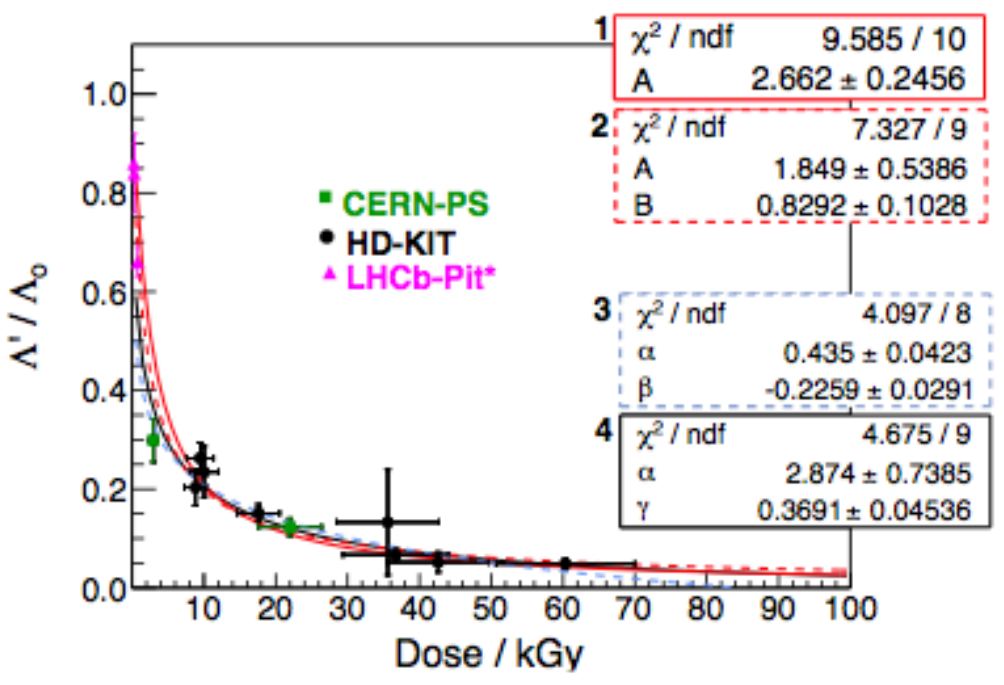

(b) Linear $\mathrm{x}$-axis

Figure 3.16: The combined attenuation length data shown with statistical errors versus dose from three fibre irradiation studies and fits to 4 models. Model 1 assumes a linear damage with dose effect $\left(\Lambda^{\prime}(D) / \Lambda_{0}=1 /(1+(D / A))\right)$. Model 2 assumes a power law function $\left(\Lambda^{\prime}(D) / \Lambda_{0}=\right.$ $\left.1 /\left(1+(D / A)^{B}\right)\right)$. Model 3 is the logarithmic function $\Lambda^{\prime}(D) / \Lambda_{0}=\alpha+\beta \log (D)$. Model 4 has an exponential-like behaviour $\left(\Lambda^{\prime}(\right.$ Dose $) / \Lambda_{0}=\exp \left((D / \alpha)^{\gamma}\right)$. The LHCb-Pit data are not yet included in these fits as they likely have much larger systematic errors that are currently being determined. 


\section{Irradiation effects in fibres}

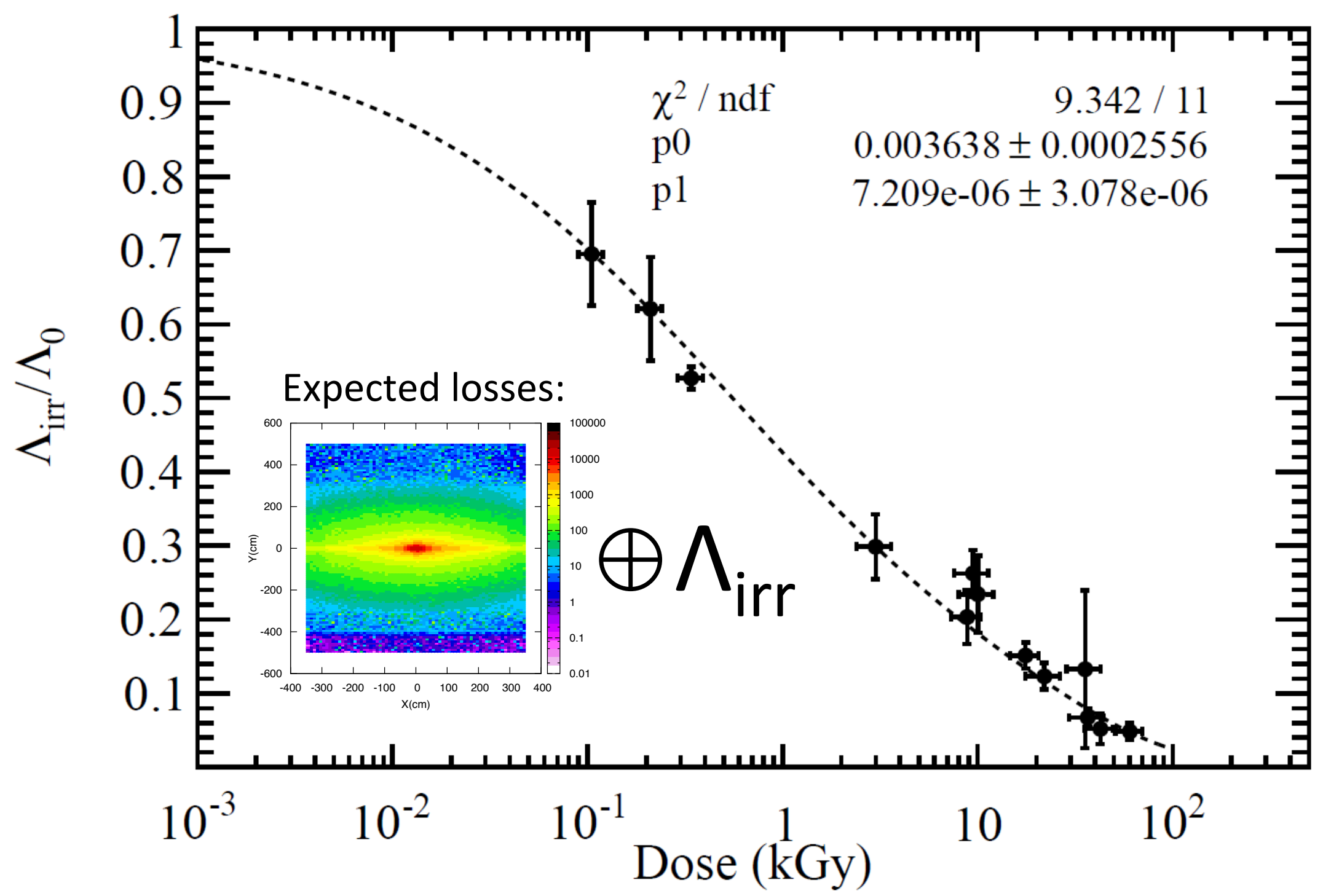




\section{KETEK Microcell Construction}
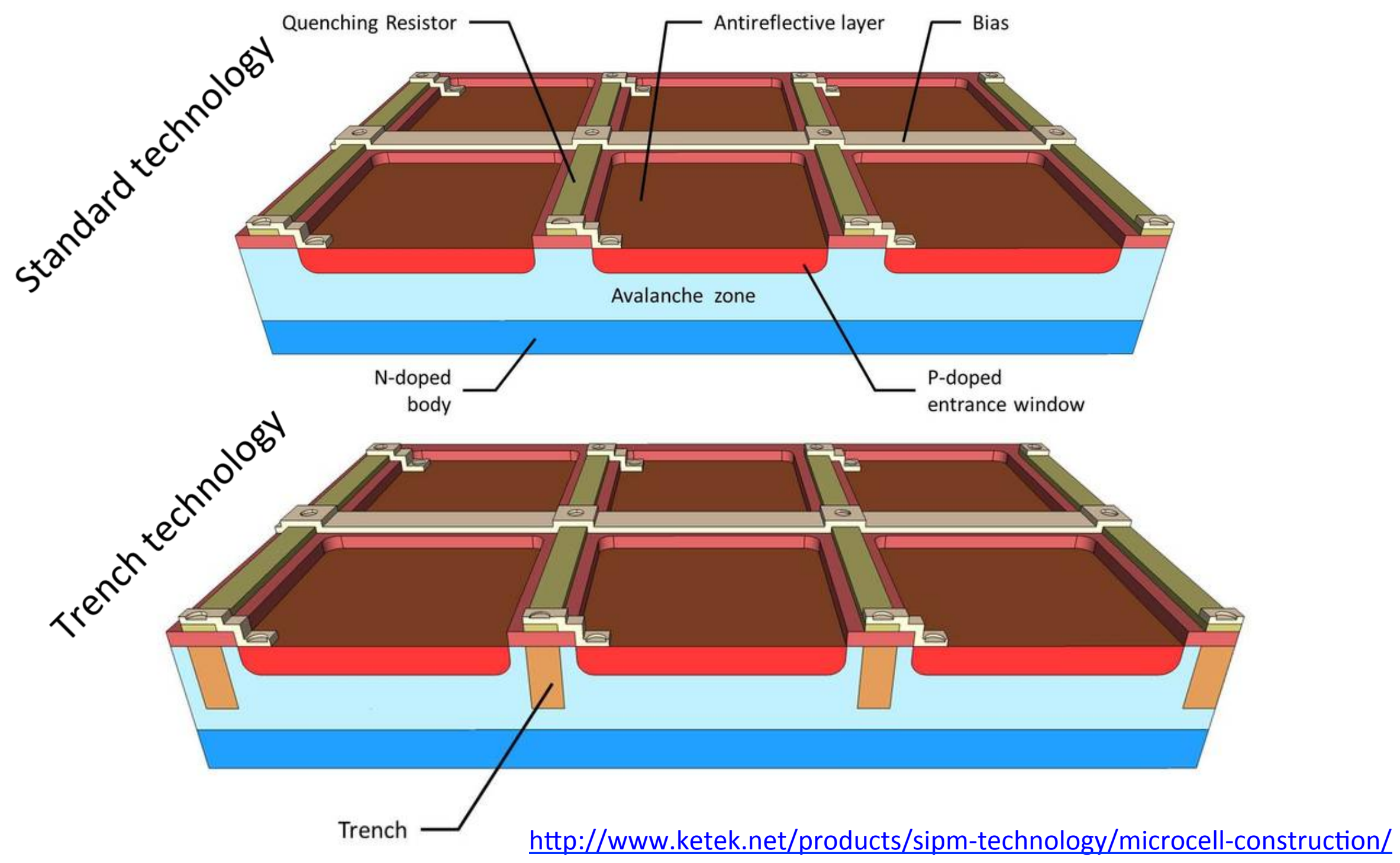


\section{Dark noise}
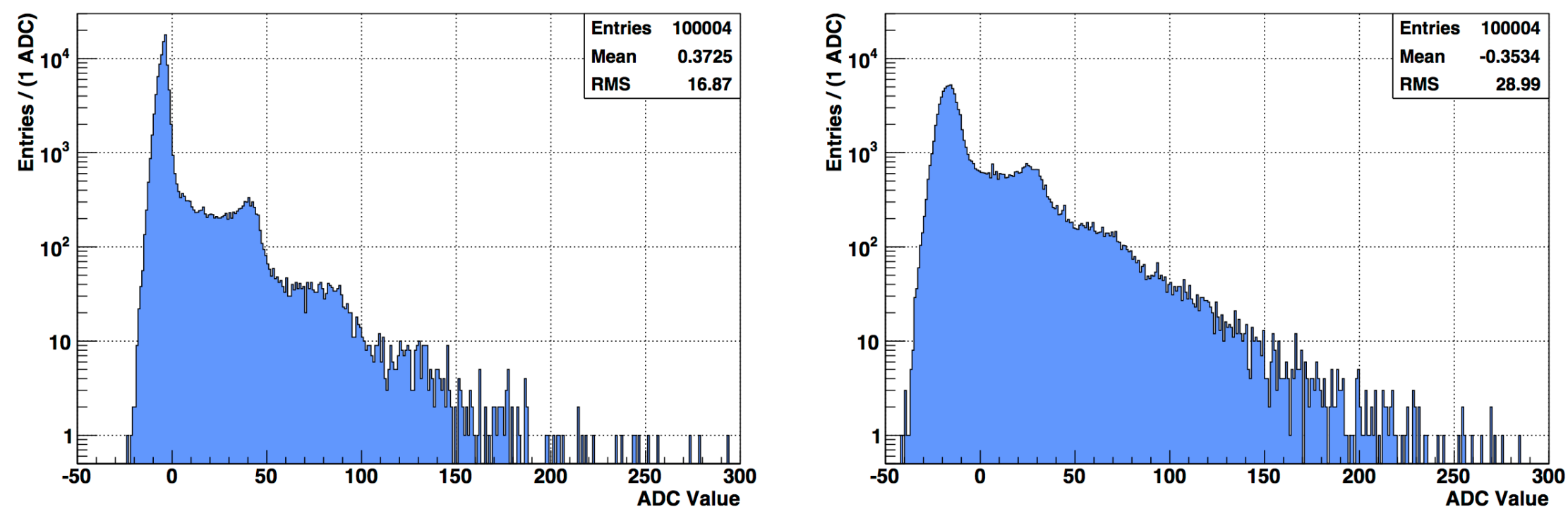

- Standard hamamatsu.

- Non-irradiated.

- $2 \times 10^{11} \mathrm{n}_{\mathrm{eq}} / \mathrm{cm}^{2}$.

- Nominal voltage.

- Nominal voltage.

- Temperature: $-60^{\circ} \mathrm{C}$.

- Temperature: $25^{\circ} \mathrm{C}$.

- Ratio between $1^{\text {st }}$ and $2^{\text {nd }}$ p.e. peaks is same.

- Cross-talk unchanged. 


\section{Annealing in SiPMs}

- Multi-channel hamamatsu arrays without trenches.

- Irradiated with neutrons up to $50 \mathrm{fb}^{-1}$.

- Dark current increases linearly with fluence.

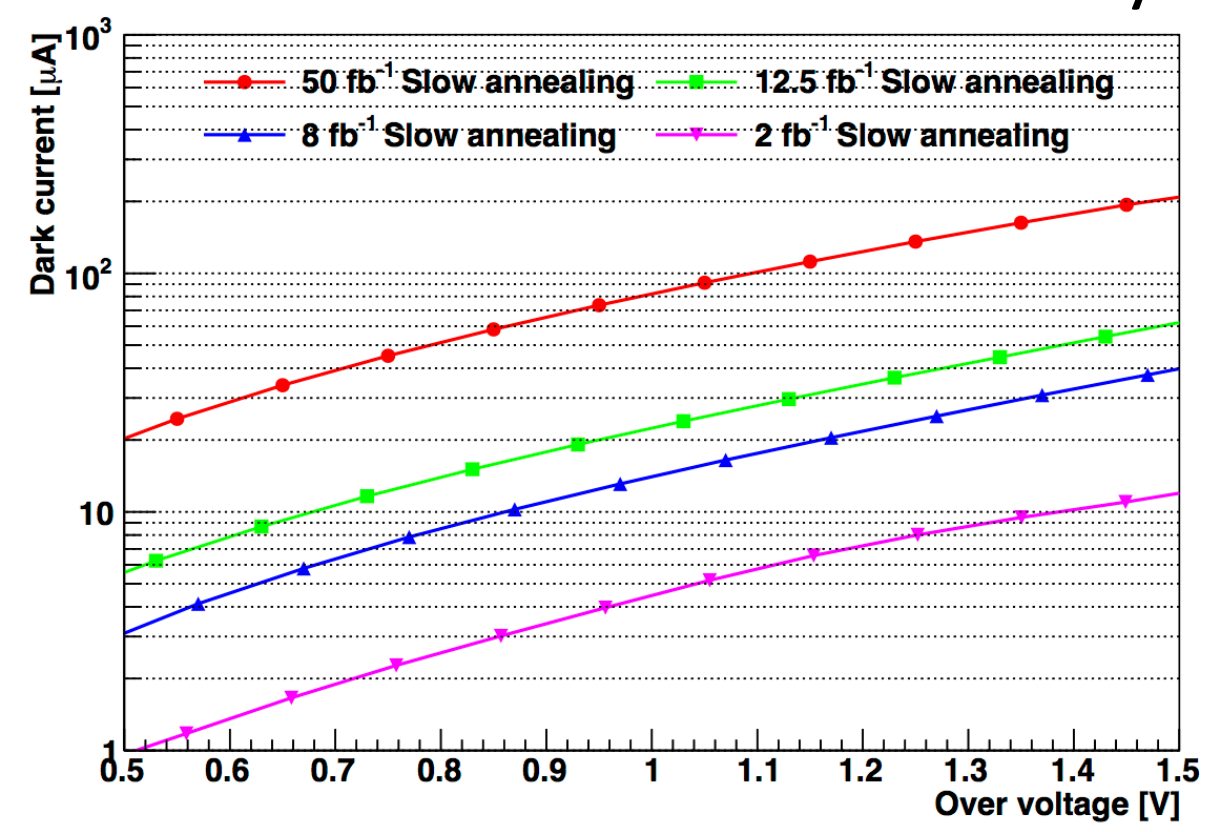

- Hamamatsu with trenches.

- Different annealing scenarios.

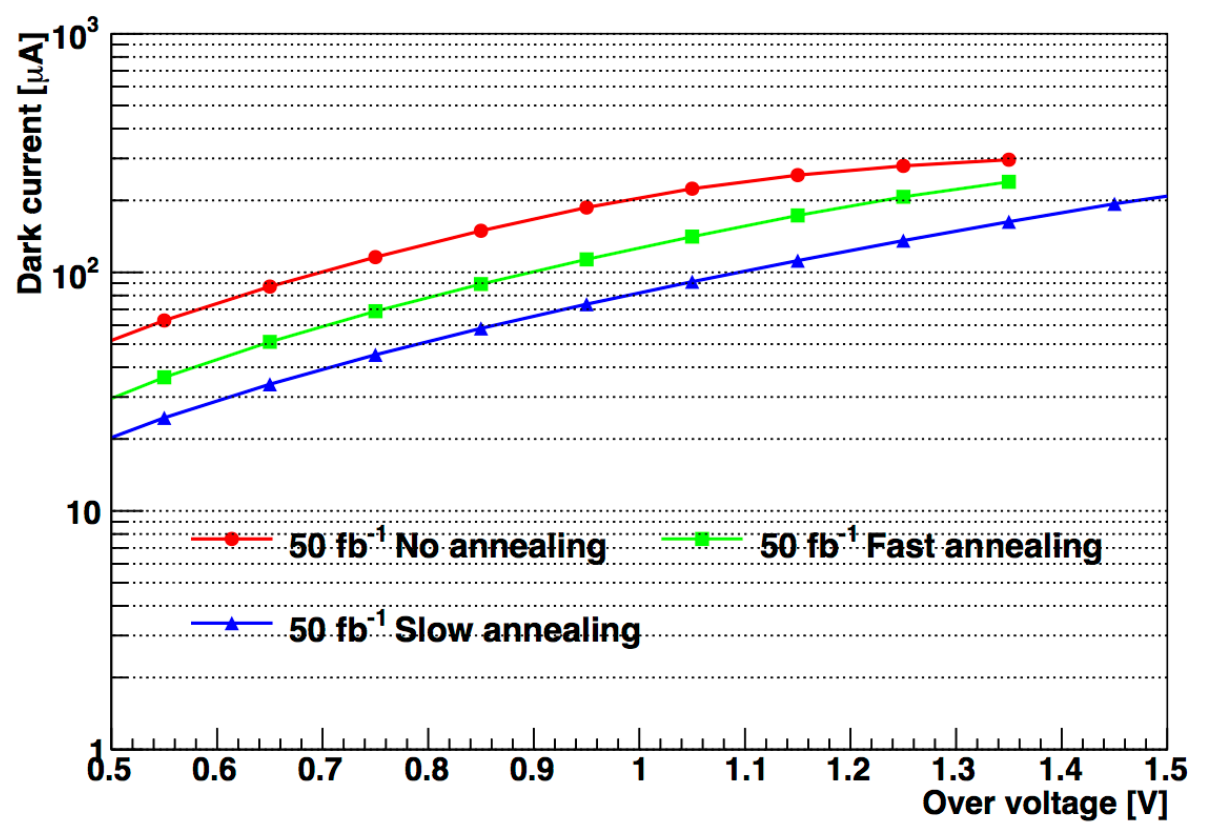




\section{Summary of SiPM measurements}

\begin{tabular}{|c|c|c|c|c|c|}
\hline Detector type & H. S10262-11-050C & $\begin{array}{c}\text { H. with trench } \\
\text { (2013) }\end{array}$ & $\begin{array}{l}\text { K. W1C2, with } \\
\text { trench }\end{array}$ & $\begin{array}{l}\text { K. W9C2, with } \\
\text { trench }\end{array}$ & $\begin{array}{c}\text { K. W7C3, double } \\
\text { trench }\end{array}$ \\
\hline Surface $\left[\mathrm{mm}^{2}\right]$ & 1 & 1 & $1 / 3$ & $1 / 3$ & $1 / 3$ \\
\hline Pixel size $\left[\mu m^{2}\right]$ & $50 \times 50$ & $50 \times 50$ & $60 \times 62.5$ & $60 \times 62.5$ & $82.5 \times 62.5$ \\
\hline $\mathrm{T}_{\mathrm{C}}[\mathrm{mV} / \mathrm{K}]$ & 56 & 53 & 15 & 26 & 22 \\
\hline Over-voltage [V] & 1.3 & 3.5 & 3.5 & 3.5 & 3.5 \\
\hline X-talk + After-pulsing & $17 \%$ & $7 \%$ & $8 \%$ & $9 \%$ & $3 \%$ \\
\hline $\mathrm{V}_{\mathrm{BD}}[\mathrm{V}]$ & 69 & 55 & 23.5 & 23.5 & 32.4 \\
\hline Gain $[e / P E]$ & $0.75 \times 10^{6}$ & $2.0 \times 10^{6}$ & $8.5 \times 10^{6}$ & $6.4 \times 10^{6}$ & $9.5 \times 10^{6}$ \\
\hline PDE @peak & $30 \%$ & $37 \%$ & $42 \%$ & $41 \%$ & $40 \%$ \\
\hline Weighted PDE integral & 1.26 & 1.61 & 1.44 & na & 1.47 \\
\hline $\begin{array}{l}\text { Scaled DCR in }[\mathrm{MHz}] \text { at } \\
-40^{\circ} \mathrm{C}, 2 \times 10^{11} \mathrm{n}_{\text {eqv }} / \mathrm{cm}^{2}, \\
1 / 3 \mathrm{~mm}^{2}\end{array}$ & 1.5 & 6 & 22 & 3.8 & 12 \\
\hline
\end{tabular}




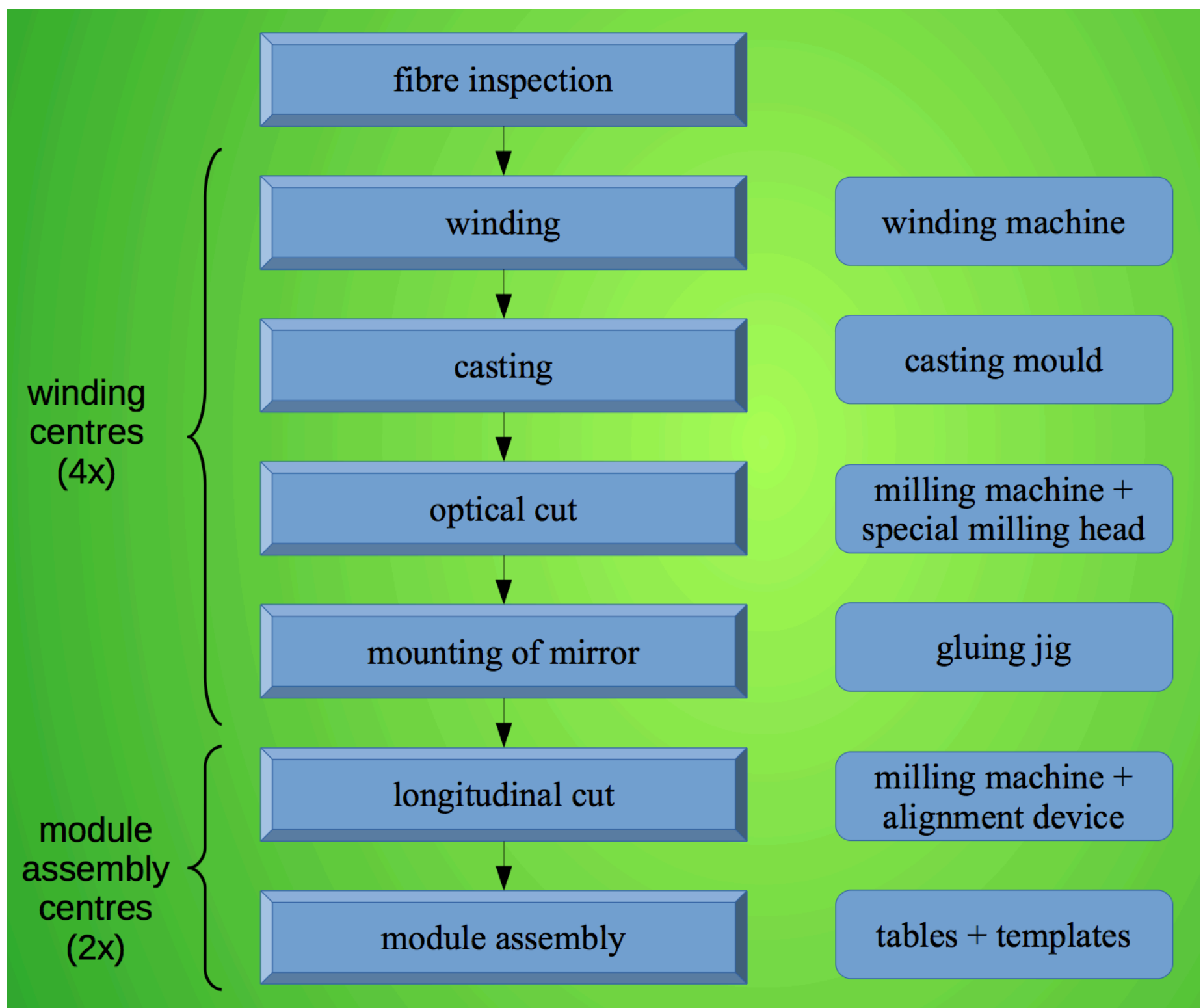

